\title{
Adjoint-based sensitivity analysis of low-order thermoacoustic networks using a wave-based approach
}

\author{
José G. Aguilar ${ }^{\mathrm{a}, *}$, Luca Magri ${ }^{\mathrm{a}, \mathrm{b}}$, Matthew P. Juniper ${ }^{\mathrm{a}}$ \\ ${ }^{a}$ Department of Engineering, University of Cambridge, Trumpington Street, Cambridge CB2 1PZ, UK \\ ${ }^{b}$ Center for Turbulence Research, Stanford University, USA
}

\begin{abstract}
Strict pollutant emission regulations are pushing gas turbine manufacturers to develop devices that operate in lean conditions, with the downside that combustion instabilities are more likely to occur. Methods to predict and control unstable modes inside combustion chambers have been developed in the last decades but, in some cases, they are computationally expensive. Sensitivity analysis aided by adjoint methods provides valuable sensitivity information at a low computational cost. This paper introduces adjoint methods and their application in wave-based low order network models, which are used as industrial tools, to predict and control thermoacoustic oscillations. Two thermoacoustic models of interest are analysed. First, in the zero Mach number limit, a nonlinear eigenvalue problem is derived, and continuous and discrete adjoint methods are used to obtain the sensitivities of the system to small modifications. Sensitivities to base-state modification and feedback devices are presented. Second, a more general case with non-zero Mach number, a moving flame front and choked outlet, is presented. The influence of the entropy waves on the computed sensitivities is shown.
\end{abstract}

Keywords: Thermoacoustic stability, adjoint methods, sensitivity analysis, network models, nonlinear eigenvalue problems

\section{Introduction}

The F-1 engines used in the Saturn V rockets were the subject of an expensive, but ultimately successful, attempt to mitigate combustion oscillations. More than 3200 full-scale tests were required [1]. Today, this cost would be prohibitive, which demonstrates the need for robust analytical tools to predict the onset of thermoacoustic oscillations and methods to control them.

Network models using wave-based approaches have been widely used in thermoacoustics [2, 3, 4]. As described by Dowling and Stow [5] a thermoacoustic network is a collection of acoustic elements such as ducts, plenums, combustors, boundaries, and a combustion zone, which is normally assumed to be compact. The elements' mean flow quantities are often considered to be homogeneous in each network element. Both mean flow quantities and fluctuations are related across elements by jump relations for the mass, momentum, and energy.

\footnotetext{
${ }^{*}$ Corresponding author

Email address: jga28@cam.ac.uk (José G. Aguilar)
} 
The primary objective of combining adjoint methods with stability analysis is to calculate the eigenvalues and their sensitivities to small modifications to the system, which can be caused by a variation of a parameter or the introduction of a feedback device [see e.g. 6, 7].

In thermoacoustics, nonlinear adjoint looping was used by Juniper [8] to find the smallest initial perturbation that could cause triggering of self-sustained oscillations in an electrically heated Rijke tube. The first application of adjoints in eigenvalue sensitivity analysis was performed by Magri and Juniper [9], who modelled a time-delayed thermoacoustic system in low Mach number conditions. Using Galerkin methods, they studied the eigenvalue sensitivity to (i) any of the parameters of the system (base state sensitivity) and (ii) generic passive control devices (feedback sensitivity, also known as structural sensitivity). One outcome was finding that a fine mesh in the second half of the tube would help to stabilize the system. This was tested experimentally by Rigas et al. [10], who measured the growth rate and the frequency shift in the presence of the passive control device. The growth rate shift was predicted accurately by adjoint analysis applied to the model. There was, however, some discrepancy in the frequency shift, which was due to limitations of the thermoacoustic model, rather than the sensitivity analysis.

Wave-based methods produce a nonlinear eigenvalue problem of the form $[11,12,13]$ :

$$
\mathrm{L}(s, \mathbf{p}) \boldsymbol{q}=\mathbf{0},
$$

where $s$ is the eigenvalue and $\mathbf{p}$ are the parameters of the system such as the reflection coefficients, time delays and heat source parameters. The adjoint function can be defined by means of a bilinear form $[\cdot, \cdot]$ such that for arbitrary $\boldsymbol{a}, \boldsymbol{b}$ :

$$
[\boldsymbol{a}, \mathrm{L} \boldsymbol{b}]-\left[\mathrm{L}^{+} \boldsymbol{a}, \boldsymbol{b}\right]=\text { constant }
$$

where $\mathrm{L}^{+}$is the adjoint operator. An operator $\mathrm{L}$ is said to be normal if its eigenfunctions $\boldsymbol{q}$ are orthogonal, or equivalently, if $\mathrm{LL}^{+}=\mathrm{L}^{+} \mathrm{L}$. Clearly, an operator is normal if it is self adjoint, i.e. $\mathrm{L}^{+}=\mathrm{L}$. The equations governing duct acoustics, without considering boundary conditions, obey the wave equation and are self-adjoint [see e.g. 14]. Nicoud et al. [15] demonstrated that thermoacoustic eigenfunctions are not orthogonal to each other, meaning that thermoacoustic systems are non-normal. Wieczorek et al. [16] showed that non-normal effects in thermoacoustics increase with the mean flow velocity. Therefore, with a mean flow, thermoacoustic systems are expected to be even less normal.

Depending on the sensitivity information desired, the operator, L, needs to be perturbed. Two different types of perturbation are defined:

- when the parameters $\mathbf{p}$ are perturbed, the resulting sensitivity is named base state sensitivity;

- when the system is perturbed by adding a small feedback mechanism, which is linearly proportional to one of the state variables of vector $\boldsymbol{q}$, the resulting sensitivity is called feedback sensitivity (also known as structural sensitivity in Giannetti and Luchini [17] and Magri and Juniper [9]). Feedback mechanisms that cause mass addition, momentum addition, and/or energy addition are considered. 
In this paper we extend adjoint-based sensitivity analysis to wave-based thermoacoustic models, which produce a nonlinear eigenvalue problem. Throughout this study, we focus on first-order perturbations. Higher-order perturbation studies have been performed by Magri [12], Magri et al. [13, 18], Mensah and Moeck [19], Silva et al. [20] but are not considered further here. First, we consider a zero-Mach number thermoacoustic system to show the symmetries between the direct and adjoint eigenfunctions, which are harder to see when the mean flow and entropy waves are included. The sensitivities are calculated using both continuous and discrete adjoint approaches, and the computational/physical advantages and disadvantages of these two methods are discussed. In the second part of this paper, the methods are extended to include a mean flow, a moving flame front, and a choked outlet in a more realistic combustor model. The paper ends with a concluding discussion.

\section{Thermoacoustic model with zero mean flow}

A one dimensional network model composed of a duct of length $L_{n}$ with a compact heat source located at $x=b$ is considered. The model assumes homogeneous properties along each segment, hence the heat source splits the domain into two segments as shown in Fig. 1. Each segment is governed by a similar set of equations, which are connected by the jump conditions established by the heat source.

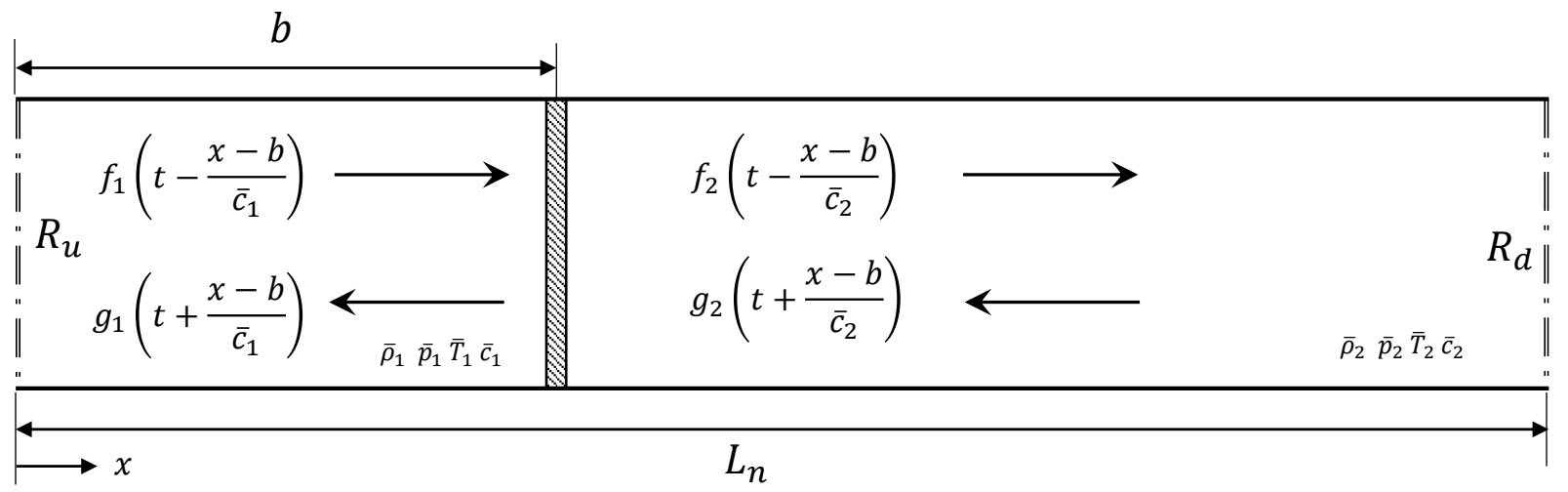

Figure 1: A simple network model. The heat source located at $x=b$ divides the duct into two segments. The arrows represent the travelling waves moving at the local speed of sound, which are labelled $f_{1}$ and $g_{1}$ upstream of the heat source and $f_{2}$ and $g_{2}$ downstream. The waves' reflection coefficients at each end are $R_{u}$ and $R_{d}$.

\subsection{Governing Equations}

The governing equations for the ducts of the thermoacoustic system are given by the continuity, momentum, and the energy equations, neglecting viscosity and heat conduction:

$$
\begin{gathered}
\frac{\partial \rho}{\partial t}+u \frac{\partial \rho}{\partial x}+\rho \frac{\partial u}{\partial x}=0, \\
\rho \frac{\partial u}{\partial t}+\rho u \frac{\partial u}{\partial x}+\frac{\partial p}{\partial x}=0, \\
\frac{\partial p}{\partial t}+u \frac{\partial p}{\partial x}+\gamma p \frac{\partial u}{\partial x}=0 .
\end{gathered}
$$


For small Helmholtz numbers, the heat source is regarded as a discontinuity in the flow parameters, thus requiring a set of jump conditions. These relations are derived from Eqs. (3) assuming that there is no accumulation of mass, momentum, or energy at the source and integrating from one side of the heat source $\left(x=b^{-}\right)$to the other $\left(x=b^{+}\right)$:

$$
\begin{gathered}
{[\rho u]_{b^{-}}^{b^{+}}=0,} \\
{\left[p+\rho u^{2}\right]_{b^{-}}^{b^{+}}=0,} \\
{\left[\frac{\gamma}{(\gamma-1)} p u+\frac{1}{2} \rho u^{3}\right]_{b^{-}}^{b^{+}}=q,}
\end{gathered}
$$

where $q$ is the heat release rate per unit area. Linearising the governing equations around the mean flow parameters (i.e., $p(x, t)=\bar{p}+p^{\prime}(x, t)$ ) and assuming a zero Mach number limit (i.e $\bar{u}=0)$ provides the steady base flow equations and the time-varying fluctuation equations. The steady base flow equations under this assumption are reduced to: $\mathrm{d} \bar{p} / \mathrm{d} x=0$ and $[\bar{p}]_{b^{-}}^{b^{+}}=0$, which implies that the pressure is continuous everywhere in the duct. Furthermore, if $\bar{u}=0$ then Eq. (4c) can only be satisfied if $\bar{q}=0$, because any heat added is not convected away. Therefore we specify $\Delta \bar{T}$ instead of $\bar{q}$. The fluctuation equations reduce to:

$$
\begin{gathered}
\bar{\rho} \frac{\partial u^{\prime}}{\partial t}+\frac{\partial p^{\prime}}{\partial x}=0 \\
\frac{\partial p^{\prime}}{\partial t}+\gamma \bar{p} \frac{\partial u^{\prime}}{\partial x}=0
\end{gathered}
$$

with the following jump conditions:

$$
\begin{aligned}
{\left[p^{\prime}\right]_{b^{-}}^{b^{+}} } & =0 \\
{\left[u^{\prime}\right]_{b^{-}}^{b^{+}} } & =\frac{\gamma-1}{\gamma \bar{p}} \int_{b^{-}}^{b^{+}} q^{\prime} \mathrm{d} x .
\end{aligned}
$$

Note that, in the zero Mach number limit, the continuity equation and the energy equation (in terms of pressure) represent the same dynamics [5]. This follows by recasting the energy equation using the isentropic relations: $p^{\prime}=\rho^{\prime} \bar{c}^{2}$ and $\gamma \bar{p}=\bar{\rho} \bar{c}^{2}$.

The unsteady heat release is taken to be from a heated gauze. This is modelled by an $n-\tau$ model in which the heat release is proportional to the velocity upstream of the gauze with a time delay [21]:

$$
q^{\prime}(x, t)=\beta u^{\prime}\left(b^{-}, t-\tau\right) \delta(x-b),
$$

where $\beta$ is the interaction index, $\tau$ the time delay between velocity and heat release and $\delta(x-b)$ is the Dirac delta.

\subsection{Decoupling the system into Riemann invariants}

The linearised governing equations (5) form a system of coupled equations which, in matrix form and using Partial Differential Equation (PDE) notation, $\boldsymbol{U}_{t}+\mathrm{A} \boldsymbol{U}_{x}=\mathbf{0}$, are:

$$
\left[\begin{array}{l}
u^{\prime} \\
p^{\prime}
\end{array}\right]_{t}+\left[\begin{array}{cc}
0 & 1 / \bar{\rho} \\
\gamma \bar{p} & 0
\end{array}\right]\left[\begin{array}{l}
u^{\prime} \\
p^{\prime}
\end{array}\right]_{x}=\mathbf{0}
$$


This represents a hyperbolic system of equations whose solutions are plane waves. To obtain the plane wave solutions, matrix $\mathrm{A}$ is diagonalized:

$$
\mathrm{A}=S \Lambda S^{-1}=\left[\begin{array}{cc}
1 / \bar{\rho} \bar{c} & -1 / \bar{\rho} \bar{c} \\
1 & 1
\end{array}\right]\left[\begin{array}{cc}
\bar{c} & 0 \\
0 & -\bar{c}
\end{array}\right]\left[\begin{array}{cc}
\bar{\rho} \bar{c} / 2 & 1 / 2 \\
-\bar{\rho} \bar{c} / 2 & 1 / 2
\end{array}\right]
$$

By substituting Eq. (9) into Eq. (8) and pre-multiplying by $S^{-1}$ we obtain:

$$
S^{-1} \boldsymbol{U}_{t}+\Lambda S^{-1} \boldsymbol{U}_{x}=\mathbf{0} .
$$

Then, by using $\boldsymbol{V}=S^{-1} \boldsymbol{U}$ we obtain the fully decoupled system, $\boldsymbol{V}_{t}+\Lambda \boldsymbol{V}_{x}=\mathbf{0}$, which reads:

$$
\left[\begin{array}{l}
\mathcal{U} \\
\mathcal{P}
\end{array}\right]_{t}+\left[\begin{array}{cc}
\bar{c} & 0 \\
0 & -\bar{c}
\end{array}\right]\left[\begin{array}{l}
\mathcal{U} \\
\mathcal{P}
\end{array}\right]_{x}=\mathbf{0}
$$

The vector $\boldsymbol{V}=[\mathcal{U}, \mathcal{P}]^{T}$ represents the Riemann invariants of the system (travelling waves). Now the system has become a set of two wave equations, with solutions:

$$
\begin{array}{ll}
\mathcal{U}(x, t)=f\left(t-\frac{x}{\bar{c}}\right) & \text { forward travelling wave, } \\
\mathcal{P}(x, t)=g\left(t+\frac{x}{\bar{c}}\right) & \text { backward travelling wave. }
\end{array}
$$

The solutions to the original variables are recovered using $\boldsymbol{U}=S \boldsymbol{V}$. According to Fig. 1 the domain is split into two sections. The wave-form solutions are centred at $x=b$, and labelled with subscripts 1 if they are located upstream of the heating gauze and 2 if they are downstream. The pressure and velocity fluctuations become:

$$
\begin{aligned}
& p^{\prime}(x, t)= \begin{cases}f_{1}\left(t-\frac{x-b}{\bar{c}_{1}}\right)+g_{1}\left(t+\frac{x-b}{\bar{c}_{1}}\right) & \text { if } x<b \\
f_{2}\left(t-\frac{x-b}{\bar{c}_{2}}\right)+g_{2}\left(t+\frac{x-b}{\bar{c}_{2}}\right) & \text { if } x>b,\end{cases} \\
& u^{\prime}(x, t)= \begin{cases}\frac{1}{\bar{\rho}_{1} \bar{c}_{1}}\left(f_{1}\left(t-\frac{x-b}{\bar{c}_{1}}\right)-g_{1}\left(t+\frac{x-b}{\bar{c}_{1}}\right)\right) & \text { if } x<b \\
\frac{1}{\bar{\rho}_{2} \bar{c}_{2}}\left(f_{2}\left(t-\frac{x-b}{\bar{c}_{2}}\right)-g_{2}\left(t+\frac{x-b}{\bar{c}_{2}}\right)\right) & \text { if } x>b .\end{cases}
\end{aligned}
$$

\subsection{Nonlinear eigenvalue problem}

First, we Laplace-transform the unsteady variables, i.e., $g_{1}=G_{1} \mathrm{e}^{s t}$ (and similarly transform $\left.f_{2}\right)$. The boundary conditions are set by means of the upstream, $R_{u}(s)$, and downstream, $R_{d}(s)$, reflection coefficients. Then we match the values of $F_{1}$ and $G_{1}$, and $F_{2}$ and $G_{2}$ at $x=b$, after reflection off the boundaries:

$$
\begin{array}{ll}
F_{1}(s)=R_{u}(s) G_{1}(s) \mathrm{e}^{-s \tau_{u}} & \tau_{u}=2 b / \bar{c}_{1}, \\
G_{2}(s)=R_{d}(s) F_{2}(s) \mathrm{e}^{-s \tau_{d}} & \tau_{d}=2\left(L_{n}-b\right) / \bar{c}_{2},
\end{array}
$$

We can therefore express the linearised jump conditions (6) in matrix form:

$$
\left[\begin{array}{cc}
-1-R_{u} \mathrm{e}^{-s \tau_{u}} & 1+R_{d} \mathrm{e}^{-s \tau_{d}} \\
\left(1-R_{u} \mathrm{e}^{-s \tau_{u}}\right)\left(1+\frac{\gamma-1}{\gamma \bar{p}_{1}} \beta \mathrm{e}^{-s \tau}\right) & \frac{\bar{c}_{2}}{\bar{c}_{1}}\left(1-R_{d} \mathrm{e}^{-s \tau_{d}}\right)
\end{array}\right]\left[\begin{array}{c}
G_{1} \\
F_{2}
\end{array}\right]=\left[\begin{array}{l}
0 \\
0
\end{array}\right],
$$


which can be written as $\mathrm{L}(s) \hat{\boldsymbol{q}}=\mathbf{0}$. This problem is a nonlinear eigenvalue problem in $s$. The eigenvalues $\left(s_{j}=\lambda_{j}+i \omega_{j}\right)$ are computed by setting the determinant of $\mathrm{L}$ to 0 . Once the eigenvalues are known, the eigenvectors $\hat{\boldsymbol{q}}_{\boldsymbol{j}}$, and subsequently the mode shapes $(\hat{p}, \hat{u})$, can be computed.

Using a wave-based formulation, adjoint-based sensitivity analysis will be performed using two different approaches: discrete and continuous. For each method the adjoint equations and eigenvalue drift formulae will be derived for the sensitivity analysis.

\subsection{Continuous adjoint approach}

Transforming the governing equations (5) and jump conditions (6) into the frequency domain using $u^{\prime}=\hat{u} \mathrm{e}^{s t}$ (and similar for $p^{\prime}$ ) leads to:

$$
\begin{aligned}
& E_{1} \equiv s \bar{\rho} \hat{u}+\frac{\mathrm{d} \hat{p}}{\mathrm{~d} x}=0, \\
& E_{2} \equiv s \hat{p}+\gamma \bar{p} \frac{\mathrm{d} \hat{u}}{\mathrm{~d} x}=0,
\end{aligned}
$$

and

$$
\begin{aligned}
& J_{1} \equiv[\hat{p}]_{b^{-}}^{b^{+}}=0, \\
& J_{2} \equiv\left[\frac{\gamma \bar{p}}{\gamma-1} \hat{u}\right]_{b^{-}}^{b^{+}}-\beta \hat{u}\left(b^{-}\right) \mathrm{e}^{-s \tau}=0 .
\end{aligned}
$$

For the set of governing equations defined in $x \in\left[0, b^{-}\right) \cup\left(b^{+}, L_{n}\right]$ there exists a corresponding set of adjoint variables $\left(\hat{u}^{+}(x), \hat{p}^{+}(x)\right)$. Similarly, for the set of jump conditions defined only at $x=b$, there exists a corresponding set of adjoint variables $\left(\hat{g}^{+}, \hat{h}^{+}\right)$. It is convenient to define the following inner products:

$$
\begin{aligned}
\text { for functions } a(x), b(x): & \langle a(x), b(x)\rangle & =\int_{0}^{b^{-}} a(x)^{*} b(x) \mathrm{d} x+\int_{b^{+}}^{L_{n}} a(x)^{*} b(x) \mathrm{d} x, \\
\text { for non-spatial functions } a, b: & \{a, b\} & =a^{*} b,
\end{aligned}
$$

where the ${ }^{*}$ denotes complex conjugation. Using the direct equations $(18,19)$ and the adjoint variables, we create a Lagrangian functional [22]:

$$
\mathcal{L} \equiv s-\left\langle\hat{u}^{+}, E_{1}\right\rangle-\left\langle\hat{p}^{+}, E_{2}\right\rangle-\left\{\hat{g}^{+}, J_{1}\right\}-\left\{\hat{h}^{+}, J_{2}\right\} .
$$

To determine the sensitivity functions of the eigenvalue following a Lagrangian approach [23], any first variation of the Lagrangian with respect to a variable $\xi$ must be equal to 0 , for any arbitrary value of $\delta \xi$ :

$$
\frac{\partial \mathcal{L}}{\partial \xi} \delta \xi \equiv \lim _{\epsilon \rightarrow 0}\left(\frac{\mathcal{L}(\xi+\epsilon \delta \xi)-\mathcal{L}(\xi)}{\epsilon}\right)=0 .
$$

For this problem, there are three sets of variables of interest: the direct variables $(\hat{u}, \hat{p})$, the adjoint variables $\left(\hat{u}^{+}, \hat{p}^{+}, \hat{g}^{+}, \hat{h}^{+}\right)$and the eigenvalue $s$. The trivial cases are the derivatives of the Lagrangian with respect to the adjoint variables, for example:

$$
\frac{\partial \mathcal{L}}{\partial \hat{u}^{+}} \delta \hat{u}^{+}=\left\langle\delta \hat{u}^{+}, E_{1}\right\rangle=0 \Longrightarrow E_{1}=0
$$


which give the direct Eqs. (18) and (19). The derivatives with respect to the direct variables require integration by parts and gives the adjoint equations:

$$
\begin{aligned}
-s^{*} \bar{\rho} \hat{u}^{+}+\gamma \bar{p} \frac{\mathrm{d} \hat{p}^{+}}{\mathrm{d} x} & =0, \\
-s^{*} \hat{p}^{+}+\frac{\mathrm{d} \hat{u}^{+}}{\mathrm{d} x} & =0,
\end{aligned}
$$

along with boundary terms. Given the unsteady heat release model, the boundary terms defined just after the heating gauze $\left(x=b^{+}\right)$relate the adjoint variables defined at the jump to those defined in the rest of the domain. After some manipulation [24] they give:

$$
\begin{aligned}
& \hat{g}^{+}=\hat{u}^{+}\left(b^{+}\right), \\
& \hat{h}^{+}=(\gamma-1) \hat{p}^{+}\left(b^{+}\right) .
\end{aligned}
$$

Similarly, the terms defined just before the heating gauze $\left(x=b^{-}\right)$provide the adjoint jump conditions:

$$
\begin{aligned}
{\left[\frac{\gamma \bar{p}}{\gamma-1} \hat{p}^{+}\right]_{b^{-}}^{b^{+}}+\beta \hat{p}^{+}\left(b^{+}\right) \mathrm{e}^{-s^{*} \tau} } & =0, \\
{\left[\hat{u}^{+}\right]_{b^{-}}^{b^{+}} } & =0 .
\end{aligned}
$$

Finally, boundary terms defined either at the inlet or outlet relate the direct boundary conditions to the adjoint boundary conditions:

$$
\hat{u}^{+*} \hat{p}+\hat{p}^{+*} \gamma \bar{p} \hat{u}=0
$$

The last quantity of interest in the Lagrangian is the eigenvalue. The Lagrangian's derivative with respect to the eigenvalue yields the normalization condition:

$$
\left\langle\hat{u}^{+}, \bar{\rho} \hat{u}\right\rangle+\left\langle\hat{p}^{+}, \hat{p}\right\rangle+\left\{\hat{h}^{+}, \tau \beta \hat{u}\left(b^{-}\right) \mathrm{e}^{-s \tau}\right\}=1 .
$$

\subsubsection{Adjoint eigenvalue problem and sources of non-self adjointness}

The adjoint equations of (5) can be found by including an integration over time in the technique described in section 2.4:

$$
\begin{aligned}
\bar{\rho} \frac{\partial u^{+}}{\partial t}+\gamma \bar{p} \frac{\partial p^{+}}{\partial x} & =0, \\
\frac{\partial p^{+}}{\partial t}+\frac{\partial u^{+}}{\partial x} & =0 .
\end{aligned}
$$

By comparing the sign of the first terms in Eqs. (24) and (29), it can be seen that, in this formulation, the Laplace transform $p^{+}(x, t)=\hat{p}^{+}(x) \mathrm{e}^{-s^{*} t}$ should be used for the adjoint equations. We can now decouple the system following a similar approach as in $\S 2.2$. We build a system of the form $\boldsymbol{U}_{t}^{+}+\mathrm{A}^{+} \boldsymbol{U}_{x}^{+}=\mathbf{0}$ that reads:

$$
\left[\begin{array}{l}
u^{+} \\
p^{+}
\end{array}\right]_{t}+\left[\begin{array}{cc}
0 & \gamma \bar{p} / \bar{\rho} \\
1 & 0
\end{array}\right]\left[\begin{array}{l}
u^{+} \\
p^{+}
\end{array}\right]_{x}=\mathbf{0} .
$$


Then matrix $\mathrm{A}^{+}$is diagonalized:

$$
\mathrm{A}^{+}=S^{+} \Lambda^{+} S^{+-1}=\left[\begin{array}{cc}
\bar{c} & -\bar{c} \\
1 & 1
\end{array}\right]\left[\begin{array}{cc}
\bar{c} & 0 \\
0 & -\bar{c}
\end{array}\right]\left[\begin{array}{cc}
1 /(2 \bar{c}) & 1 / 2 \\
-1 /(2 \bar{c}) & 1 / 2
\end{array}\right]
$$

We note that matrix $\Lambda^{+}=\Lambda$. By substituting $\mathrm{A}^{+}$into Eq. (30) and pre-multiplying by $S^{+^{-1}}$ we obtain:

$$
S^{+-1} \boldsymbol{U}^{+}{ }_{t}+\Lambda S^{+-1} \boldsymbol{U}^{+}{ }_{x}=\mathbf{0} .
$$

Then, by using $\boldsymbol{V}^{+}=S^{+-1} \boldsymbol{U}^{+}$, we obtain the fully decoupled system, $\boldsymbol{V}^{+}{ }_{t}+\Lambda \boldsymbol{V}^{+}{ }_{x}=\mathbf{0}$, which is:

$$
\left[\begin{array}{l}
\mathcal{U}^{+} \\
\mathcal{P}^{+}
\end{array}\right]_{t}+\left[\begin{array}{cc}
\bar{c} & 0 \\
0 & -\bar{c}
\end{array}\right]\left[\begin{array}{l}
\mathcal{U}^{+} \\
\mathcal{P}^{+}
\end{array}\right]_{x}=\mathbf{0}
$$

Again, the vector $\boldsymbol{V}^{+}=\left[\mathcal{U}^{+}, \mathcal{P}^{+}\right]^{T}$ represents the Riemann invariants of the adjoint system. Since the diagonal matrix $\Lambda$ is the same for the direct and adjoint equations, $\boldsymbol{V}^{+}$equals $\boldsymbol{V}$. Therefore the solutions of the wave equations are the same as in the direct problem:

$$
\begin{array}{ll}
\mathcal{U}^{+}(x, t)=f^{+}\left(t-\frac{x}{\bar{c}}\right) & \text { forward travelling wave, } \\
\mathcal{P}^{+}(x, t)=g^{+}\left(t+\frac{x}{\bar{c}}\right) & \text { backward travelling wave. }
\end{array}
$$

The equations governing the ducts, without considering boundary conditions, are those for pure acoustics. Unsurprisingly, this analysis show that they are self-adjoint. An alternative demonstration exists in the zero Mach number limit by making the variable change $\psi^{+}=\gamma \bar{p} p^{+}$. The adjoint system in the time domain becomes:

$$
\begin{aligned}
\bar{\rho} \frac{\partial u^{+}}{\partial t}+\frac{\partial \psi^{+}}{\partial x} & =0 \\
\frac{\partial \psi^{+}}{\partial t}+\gamma \bar{p} \frac{\partial u^{+}}{\partial x} & =0 \\
{\left[\psi^{+}\right]_{b^{-}}^{b^{+}} } & =-\frac{\gamma-1}{\gamma \bar{p}} \beta \psi^{+}\left(b^{+}, t+\tau\right), \\
{\left[u^{+}\right]_{b^{-}}^{b^{+}} } & =0
\end{aligned}
$$

which is the same as in the direct case Eqs. (5) and (6) except for the unsteady heat release $(\beta)$ term, which has switched from the $u^{+}$jump condition to the $\psi^{+}$jump condition and now relies on $t$ at a later time, $t+\tau$. (The latter point shows that the adjoint equations naturally evolve backwards in time.) It is evident that the unsteady heat release term makes the system non-self-adjoint through the jump conditions.

After decoupling the system, we define the adjoint reflection coefficients, $R_{u}^{+}$and $R_{d}^{+}$, as for the direct case Eqs. (15) and (16). By applying Eq. (27), which links boundary terms at the inlet and outlet, we obtain:

$$
R_{u}^{+}=\frac{1}{R_{u}^{*}}
$$




$$
R_{d}^{+}=\frac{1}{R_{d}^{*}}
$$

The adjoint reflection coefficients are the inverse conjugates of the direct reflection coefficients. At first sight, this suggests that the system will be non-self-adjoint whenever $\left|R_{u}\right| \neq 1$ or $\left|R_{d}\right| \neq 1$ (and hence, non-normal as pointed out, for example, by Mangesius and Polifke [25]). However, since the Laplace variable for the adjoint problem $\left(f_{1}^{+}=F_{1}^{+} \mathrm{e}^{-s^{*} t}\right)$ is the negative of that of the direct problem $\left(f_{1}=F_{1} \mathrm{e}^{s t}\right)$, the direction of the travelling waves in the adjoint case is opposite to that in the direct case (i.e $F_{1}^{+}$corresponds to $G_{1}$ and $G_{2}^{+}$corresponds to $F_{2}$ ). This results in the following adjoint eigenvalue problem:

$$
\left[\begin{array}{cc}
-1-R_{u}^{*} \mathrm{e}^{-s^{*} \tau_{u}} & \left(1+R_{d}^{*} \mathrm{e}^{-s^{*} \tau_{d}}\right)\left(1+\frac{\gamma-1}{\gamma \bar{p}_{1}} \beta \mathrm{e}^{-s^{*} \tau}\right) \\
1-R_{u}^{*} \mathrm{e}^{-s^{*} \tau_{u}} & \bar{c}_{2}\left(1-R_{d}^{*} \mathrm{e}^{-s^{*} \tau_{d}}\right)
\end{array}\right]\left[\begin{array}{c}
F_{1}^{+} \\
G_{2}^{+}
\end{array}\right]=\mathbf{0},
$$

which can be written as $\mathrm{L}^{+}(s) \hat{\boldsymbol{q}}^{+}=\mathbf{0}$. Comparing it with the direct matrix L from Eq. (17), the unsteady heat release term has switched from the bottom-left element to the top-right element. The determinant, however, remains the same apart from the complex conjugation of the frequency-dependent terms, which arises due to the definition of the inner product in (20). Hence, we notice that the adjoint eigenvalues are the complex conjugates of the direct eigenvalues [9], but the adjoint eigenvectors are not the complex conjugate of the direct eigenvectors, due to the re-positioning of the heat release term. Note that, in the absence of the unsteady heat release, matrices $\mathrm{L}$ and $\mathrm{L}^{+*}$ are identical, and the system is self-adjoint even when the magnitudes of the reflection coefficients are not 1 .

\subsubsection{Base state and feedback sensitivities}

The eigenvalue drift associated with base state or feedback sensitivities using a continuous approach is derived through the following steps [24]:

1. From the eigenvalue problem Eq. (17) we compute the eigenvalue of interest, $s_{j}$.

2. We obtain $\hat{p}$ and $\hat{u}$ from the direct eigenvalue problem. Similarly, from the adjoint eigenvalue problem, we obtain $\hat{p}^{+}$and $\hat{u}^{+}$.

3. We normalize using Eq. (28).

4. For the feedback sensitivity only, we add a small feedback perturbation to the system. For example, for feedback of velocity into the momentum equation at position $x_{0}$ this is:

$$
E_{1}-\mathcal{F}_{u} \hat{u}(x) \delta\left(x-x_{0}\right)=0 .
$$

5. We obtain the first variation of the Lagrangian with respect to the base state variable or feedback mechanism of interest, which provides the eigenvalue drift. For the base state variable $\tau$ this is:

$$
\frac{\partial \mathcal{L}}{\partial \tau} \delta \tau=0 \Longrightarrow \delta s=-\left\{\hat{h}^{+}, \beta \hat{u}\left(b^{-}\right) \mathrm{e}^{-s \tau} s \delta \tau\right\}
$$

For base state variables such as reflection coefficients, $R_{u}$ and $R_{d}$, or geometric time delays, $\tau_{u}$ and $\tau_{d}$, the wave-form solutions (13) and (14) together with the boundary conditions (15) and (16) must be substituted into the original Eqs. (5) and (6). The equations governing 
the ducts (5) will be trivially satisfied. Hence, from the jump conditions in the frequency domain we obtain their first variations, for example:

$$
\frac{\partial \mathcal{L}}{\partial \tau_{d}} \delta \tau_{d}=0 \Longrightarrow \delta s=\left\{\hat{g}^{+}, s F_{2} R_{d} \mathrm{e}^{-s \tau_{d}} \delta \tau_{d}\right\}-\left\{\hat{h}^{+}, s F_{2} R_{d} \mathrm{e}^{-s \tau_{d}}\left(\frac{\bar{c}_{2}}{\gamma-1}\right) \delta \tau_{d}\right\} .
$$

\subsection{Discrete adjoint approach}

For the nonlinear eigenvalue problem (17) there exists an adjoint eigenvector $\left(\hat{\boldsymbol{q}}^{\dagger}\right)$ that satisfies:

$$
\left(\hat{\boldsymbol{q}}^{\dagger}\right)^{H} \mathrm{~L}(s)=\mathbf{0} .
$$

For these problems, $[12,13]$ derived the following eigenvalue drift formula:

$$
\delta s=-\frac{\left(\hat{\boldsymbol{q}}^{\dagger}\right)^{H} \delta \mathrm{L}\left(s_{j}\right) \hat{\boldsymbol{q}}}{\left(\hat{\boldsymbol{q}}^{\dagger}\right)^{H} \frac{\partial \mathrm{L}\left(s_{j}\right)}{\partial s_{j}} \hat{\boldsymbol{q}}}
$$

where the matrix $\delta \mathrm{L}$ represents the first variation of a variable in $\mathrm{L}$.

\subsubsection{Base state sensitivity}

The matrix $\delta \mathrm{L}$ for the base state variables is:

$$
\delta \mathrm{L}=\frac{\partial \mathrm{L}}{\partial R_{u}} \delta R_{u}+\frac{\partial \mathrm{L}}{\partial R_{d}} \delta R_{d}+\frac{\partial \mathrm{L}}{\partial \tau_{u}} \delta \tau_{u}+\frac{\partial \mathrm{L}}{\partial \tau_{d}} \delta \tau_{d}+\frac{\partial \mathrm{L}}{\partial \beta} \delta \beta+\frac{\partial \mathrm{L}}{\partial \tau} \delta \tau .
$$

For example, the eigenvalue drift for a generic change in the downstream reflection coefficient, $R_{d}$, is given by $\delta s / \delta R_{d}=-\left(\left(\hat{\boldsymbol{q}}^{\dagger}\right)^{H} \frac{\partial \mathrm{L}\left(s_{j}\right)}{\partial R_{d}} \hat{\boldsymbol{q}}\right) /\left(\left(\hat{\boldsymbol{q}}^{\dagger}\right)^{H} \frac{\partial \mathrm{L}\left(s_{j}\right)}{\partial s_{j}} \hat{\boldsymbol{q}}\right)$.

\subsubsection{Feedback sensitivity}

To compute the matrix $\delta \mathrm{L}$ associated with the feedback sensitivity of the system, the effects of a feedback mechanismhas needs to be considered. This is done by introducing small timevarying disturbances proportional to $p^{\prime}$ and $u^{\prime}$ into the fluctuation equations (Eq. (5)), which correspond to adding a force $\left(\mathcal{F}_{p}, \mathcal{F}_{u}\right)$ into the momentum equation or introducing heat $\left(\mathcal{Q}_{p}, \mathcal{Q}_{u}\right)$ into the energy equation. The main assumption is that these perturbations do not produce any change to the base flow parameters. The major consequence is that, to keep a wave-based model, intrinsic feedback must be introduced as a jump similar to the heat source, which in turn splits the system into four segments as shown in Fig. 2. Following the notation in Fig. 2, the set of jump conditions upstream of the heat source $(x=a)$ reads:

$$
\begin{aligned}
p_{i i}^{\prime}-p_{i}^{\prime} & =\mathcal{F}_{p_{1}} p_{i}^{\prime}+\mathcal{F}_{u_{1}} u_{i}^{\prime}, \\
\gamma \bar{p}_{1}\left(u_{i i}^{\prime}-u_{i}^{\prime}\right) & =\mathcal{Q}_{p_{1}} p_{i}^{\prime}+\mathcal{Q}_{u_{1}} u_{i}^{\prime} .
\end{aligned}
$$

The set of jump conditions downstream of the heat source $(x=c)$ reads:

$$
\begin{aligned}
p_{i v}^{\prime}-p_{i i i}^{\prime} & =\mathcal{F}_{p_{2}} p_{i v}^{\prime}+\mathcal{F}_{u_{2}} u_{i v}^{\prime} \\
\gamma \bar{p}_{2}\left(u_{i v}^{\prime}-u_{i i i}^{\prime}\right) & =\mathcal{Q}_{p_{2}} p_{i v}^{\prime}+\mathcal{Q}_{u_{2}} u_{i v}^{\prime}
\end{aligned}
$$




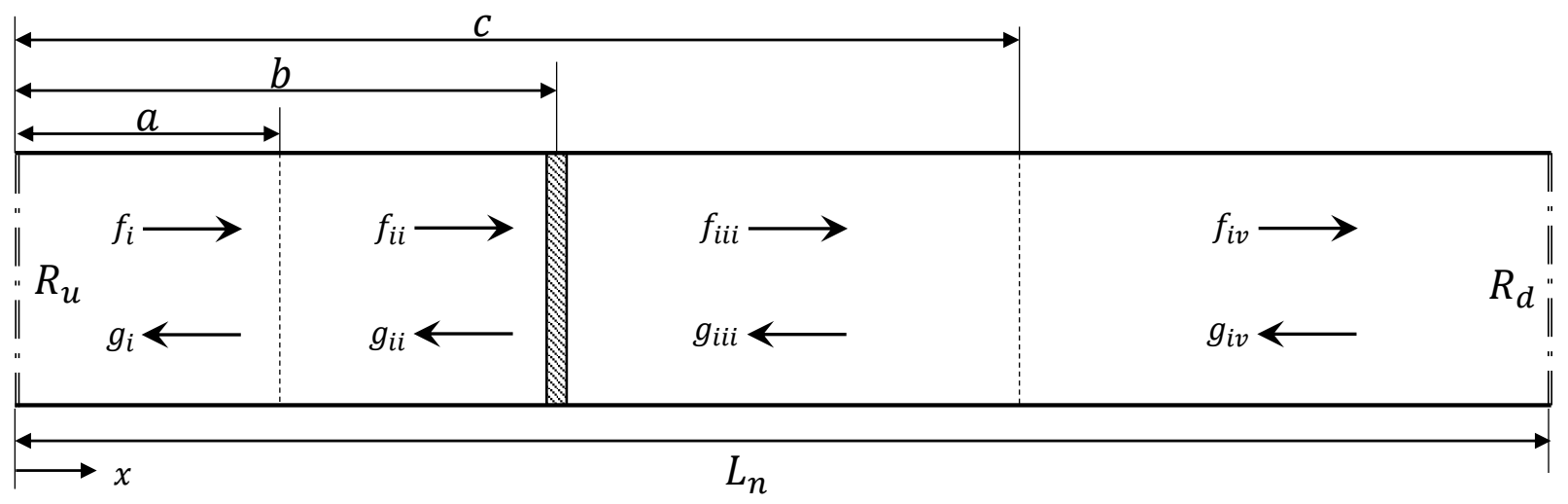

Figure 2: Network model split into 4 segments due to the presence of intrinsic feedback at $x=a$ and $x=c$. The original system can be thought of as being the heat source and the ducts from regions $i i$ and $i i i$. Since the perturbations must be introduced from the surroundings into the system (see Eqs. 44 and 45 ), the depicted wave-forms from regions $i$ and $i v$ represent the unperturbed waves $\left(f_{i}=f_{1}\right.$ and $\left.f_{i v}=f_{2}\right)$, while the wave-forms of regions $i i$ and $i i i$ represent the perturbed waves $\left(f_{i i}=f_{1}+\delta f_{1}\right.$ and $\left.f_{i i i}=f_{2}+\delta f_{2}\right)$.

Because the elements of matrix L are the coefficients for the amplitudes of wave forms $(f, g)$, the elements of matrix $\delta \mathrm{L}$ should be their first variations $(\delta f, \delta g)$ induced by the application of the perturbations $(\mathcal{F}, \mathcal{Q})$, which is: $\delta \mathrm{L} \rightarrow \delta \mathrm{L}(\mathcal{F}, \mathcal{Q})$. To get the wave forms together with their first variations (i.e $f+\delta f$ ), we solve both $2 \times 2$ systems generated by the new sets of jump conditions (Eqs. 44 and 45) for the waves in regions $i$ and iii. For example:

$$
\begin{aligned}
f_{i i}(t) & =f_{1}(t)+\frac{1}{2}\left(\mathcal{F}_{p_{1}}\left(f_{1}(t)+g_{1}\left(t-\tau_{a}\right)\right)+\frac{\mathcal{F}_{u_{1}}}{\bar{\rho}_{1} \bar{c}_{1}}\left(f_{1}(t)-g_{1}\left(t-\tau_{a}\right)\right) \cdots\right. \\
& \left.+\frac{\mathcal{Q}_{p_{1}}}{\bar{c}_{1}}\left(f_{1}(t)+g_{1}\left(t-\tau_{a}\right)\right)+\frac{\mathcal{Q}_{u_{1}}}{\bar{\rho}_{1} \bar{c}_{1}^{2}}\left(f_{1}(t)-g_{1}\left(t-\tau_{a}\right)\right)\right),
\end{aligned}
$$

where $\tau_{a}=2(b-a) / \bar{c}_{1}$. The four perturbed wave solutions have a similar structure in terms of coefficients. After applying the same boundary conditions, Laplace transforms and substitution into the original jump conditions Eq. (6) as in $\S 2.3$ we obtain the desired system:

$$
(\mathrm{L}+\delta \mathrm{L})(\hat{\boldsymbol{q}}+\boldsymbol{\delta} \hat{\boldsymbol{q}})=\mathbf{0}
$$

Matrix $\delta \mathrm{L}$ is computed in Appendix A following the above procedure. To obtain the feedback sensitivities, the eigenvalue drift is computed at several positions between the inlet and the outlet by keeping only one of the feedback elements active at a time, bearing in mind that the equations are linear and the superposition of effects holds.

\subsection{Finite-difference method}

After perturbing any of the equations or variables in the system, we re-compute the perturbed eigenvalue, $s_{j}+\delta s_{j}$, and then subtract from it the unperturbed eigenvalue, $s_{j}$. The major drawback is that, unlike adjoint methods, this requires another full computation for every perturbation. This finite-difference method is used throughout this paper to check the sensitivities provided by the adjoint equations. 


\section{Continuous adjoint vs. discrete adjoint approaches}

The main advantage of the continuous adjoint approach is that the adjoint variables are directly expressed in physical space, i.e., the adjoint spatial functions are known. This means that (i) base state and feedback sensitivities are computed as inner products between direct and adjoint variables and (ii) one can obtain good physical understanding of the spatial system's receptivity to harmonic forcing ${ }^{1}$. The main disadvantage is that the adjoint equations, boundary conditions and normalization condition, need to be derived analytically.

The main advantage of the discrete adjoint is that, as long as the the operator $\mathrm{L}$ is known, computing the base state sensitivities does not require any further implementation other than a complex transpose (see $\S 2.5 .1$ ). The main disadvantage is that, to compute the feedback sensitivity, we need to introduce the feedback perturbations by splitting the domain as in Fig. 2 . This implementation is more complicated than that of the continuous approach.

The sensitivities of the eigenvalue computed using continuous and discrete approaches are the same to machine precision, and they match the results by finite difference. Since the results are identical, the sensitivities computed in the following sections do not distinguish between the approaches used.

\section{Results for an open-ended duct with zero mean flow}

\subsection{Thermoacoustic configuration}

The variables are non-dimensionalized using the following reference scales for length, time, speed and pressure, respectively: $L_{n}, L_{n} / \bar{c}_{1}, \bar{c}_{1}$ and $\gamma \bar{p}_{1}$. The non-dimensional variables are written with a tilde, i.e., $\tilde{t}=t \cdot \bar{c}_{1} / L_{n}$. Pressure eigenfunctions for the direct and adjoint variables are normalized as $\int_{0}^{L_{n}} \hat{p}(x) \hat{p}(x)^{*} \mathrm{~d} x=1, \int_{0}^{L_{n}} \hat{p}^{+}(x) \hat{p}^{+}(x)^{*} \mathrm{~d} x=1$. We consider a Rijke tube of length $L_{n}=1 \mathrm{~m}$; dry air properties, $\gamma=1.40$ and $R=287.1 \mathrm{Jkg}^{-1} \mathrm{~K}^{-1}$; inlet pressure of $\bar{p}_{1}=101.3 \mathrm{kPa}$ and inlet temperature of $\bar{T}_{1}=300.0 \mathrm{~K}$; open ends at the inlet and outlet, $R_{u}=-1, R_{d}=-1$; jump in temperature of $\Delta \bar{T}=300.0 \mathrm{~K}$ caused by the heating gauze located at $b=0.250 \mathrm{~m}$ with interaction index $\beta=360.0 \times 10^{3} \mathrm{~ms}^{2} \mathrm{~kg}^{-1}$ and time delay $\tau=$ $1.00 \times 10^{-3} \mathrm{~s}$. Following the procedure from $\S 2.3$ we find that the dominant eigenvalue is $s_{1}=(134.1+2 \pi \times 222.2 i) \mathrm{rad} \mathrm{s}^{-1}$, in non-dimensional units: $\tilde{s}_{1}=0.3862+4.021 i$.

\subsection{Stability and sensitivity analysis}

The direct and adjoint mode shapes are shown in Fig. 3. Where the amplitude of the direct mode shape is large, the system is more observable. Where the amplitude of the adjoint mode shape is large, the system is more controllable. For this configuration, the pressure and velocity mode shapes of Figs. 3a and 3b display several important characteristics of thermoacoustic systems. The pressure is continuous at the heating gauze, with a slight change of gradient, while the velocity has a jump at the heat source, as expected from the jump conditions (6). For the governing equations in the zero mean flow case, Eq. (5), it is easy to show that when a Dirichlet

\footnotetext{
${ }^{1}$ The reason why the adjoint variables represent the receptivity of the system originates from bi-orthogonal decomposition $[7,22]$.
} 
boundary condition is imposed on $p^{\prime}$, a Neumann boundary condition is instantly imposed on $u^{\prime}$, and vice-versa. The former statement is depicted in the amplitudes of the direct mode shapes. With regards to the adjoint mode shapes, we see in Fig. 3a that the receptivity to the energy equation $\left(\hat{p}^{+}\right)$inherits the jump at the heat source location from $u^{\prime}$ and the boundary conditions from $p^{\prime}$. Additionally, note that the receptivity to the momentum equation, $\hat{u}^{+}$, is now continuous with a slight change of gradient at the heating gauze location. Although not shown here, with zero unsteady heat release the direct pressure mode shape is identical to the adjoint pressure mode shape, and the direct velocity mode shape is identical to the adjoint velocity mode shape (see Section 2.4.1).

(a)

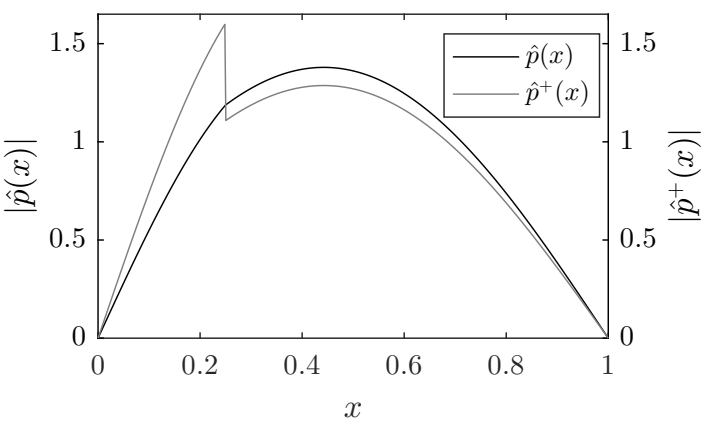

(b)

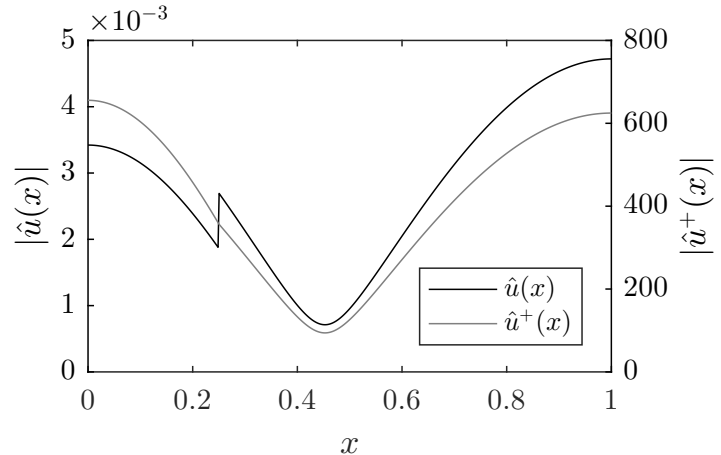

Figure 3: Mode shapes for the direct and adjoint variables. (a) and (b) show the relative amplitudes between pressure and velocity fluctuations, i.e., $\hat{p}(x)=|\hat{p}(x)| \mathrm{e}^{i \theta}$, as well as the receptivity to the energy and momentum equations respectively (computed using the continuous adjoint approach).

In a self-adjoint system, the best position for a Helmholtz resonator is where $\hat{p} \hat{p}^{*}$ is maximal, which is at the centre of the tube. In a system that it is not self-adjoint, this is no longer true. Even though the pressure is still maximum around the centre of the tube, the system is more receptive before the heating gauze location. The best location to place the Helmholtz resonator in such a system is where the amplitude of $\hat{p} \hat{p}^{+*}$ is maximum, which, in this case, is slightly upstream of the centre of the tube (Fig. 3a).

(a)

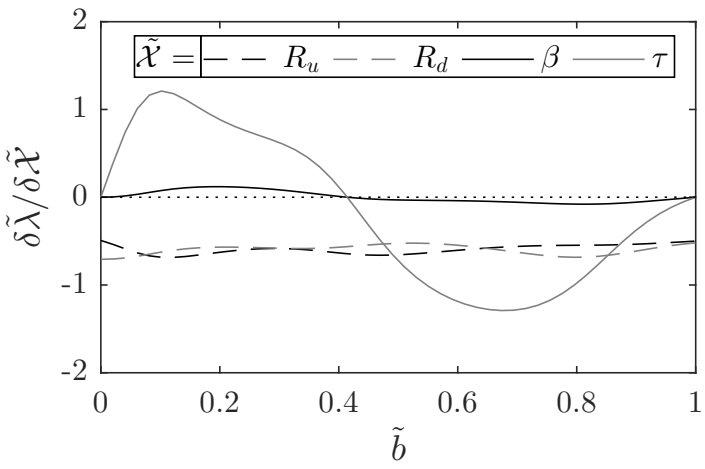

(b)

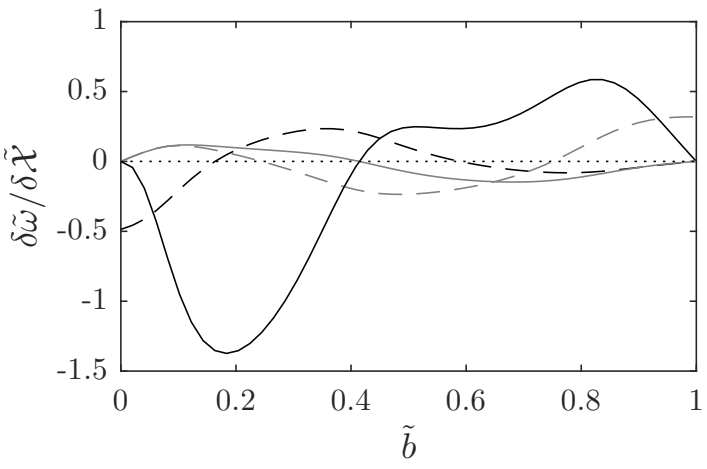

Figure 4: Growth rate shift $\delta \tilde{\lambda}$ (a) and angular frequency shift $\delta \tilde{\omega}$ (b) shifts of the base state variables, denoted by $\tilde{\mathcal{X}}$, for different positions of the heating gauze, $\tilde{b}$, along the duct length. 
The base state sensitivities for different positions of the heating gauze are presented in Fig. 4. It can be seen that any small increase (from -1) in the reflection coefficients, $R_{u}$ and $R_{d}$, makes the system more stable. This is because less acoustic energy is reflected back into the tube. The interaction index, $\beta$, has the smallest amplitude and it predicts, as expected, that a heating gauze in the second half of the duct will stabilize the system. The time delay, $\tau$, of the unsteady heat release has a great impact on the eigenvalue drift. Any increase will destabilize the system if the heating gauze is located in the first half of the duct before $\tilde{b}=0.4$. Many of these remarks can be made from physical arguments. The novelty is that, with these adjoint methods, they can be calculated cheaply by an automated process and therefore could be used in a gradient-based optimization routine.

The feedback sensitivity encompasses four feedback mechanisms, shown in Fig 5. The figure recovers similar results as those obtained using Galerkin methods, by Magri and Juniper [9] for uniform temperature and in Magri and Juniper [22] considering a jump in temperature. This achieves this paper's aim of validating the wave-based adjoint method.

(a)

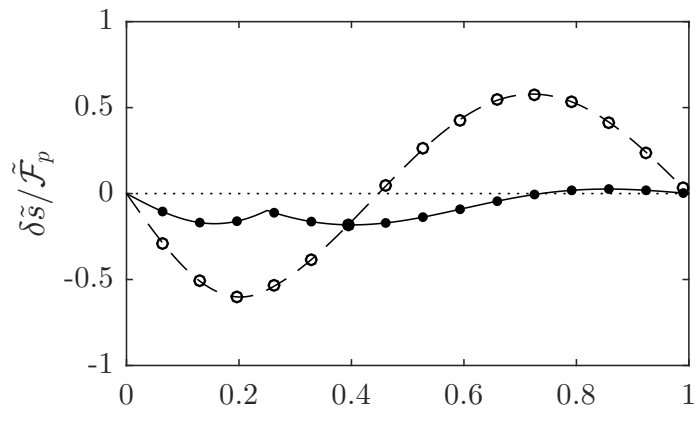

(c)

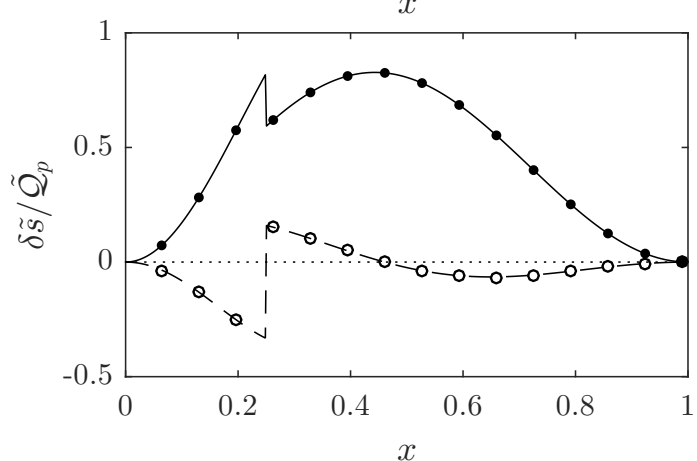

(b)

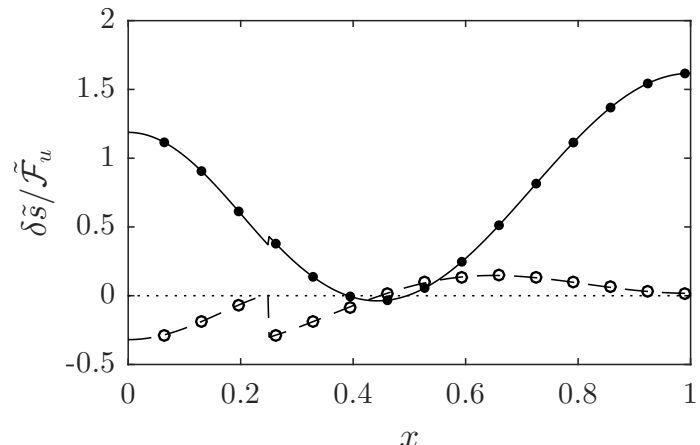

(d)

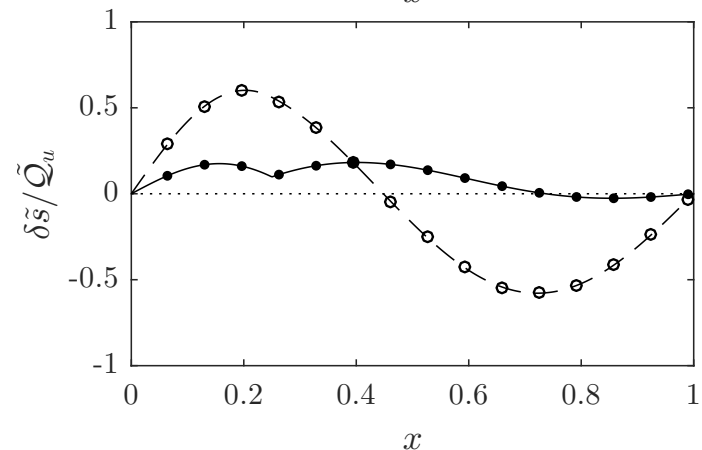

Figure 5: Growth rate shift $\delta \tilde{\lambda}$ denoted by -, •, and angular frequency shift $\delta \tilde{\omega}$ denoted by - - - , ०, for each feedback mechanism. (a) and (b) display the drifts due to forcing the momentum equation with a force proportional to pressure and velocity fluctuations respectively. Similarly (c) and (d) show the drifts due to heat addition into the system. The continuous and dashed lines (-, - -) represent one calculation (via the adjoint method) while the circles $(\bullet, \circ)$ represent 15 computations (via the finite difference method). 


\section{Thermoacoustic model with mean flow and a moving flame front}

\subsection{Governing equations}

A non-zero mean flow, $\bar{u} \neq 0$, appears in the linear Eqs. (3) as follows:

$$
\begin{gathered}
\frac{\partial \rho^{\prime}}{\partial t}+\bar{u} \frac{\partial \rho^{\prime}}{\partial x}+\bar{\rho} \frac{\partial u^{\prime}}{\partial x}=0 \\
\bar{\rho} \frac{\partial u^{\prime}}{\partial t}+\bar{\rho} \bar{u} \frac{\partial u^{\prime}}{\partial x}+\frac{\partial p^{\prime}}{\partial x}=0 \\
\frac{\partial p^{\prime}}{\partial t}+\bar{u} \frac{\partial p^{\prime}}{\partial x}+\gamma \bar{p} \frac{\partial u^{\prime}}{\partial x}=0
\end{gathered}
$$

and in the jump conditions Eq. (4). Now, however, instead of the heating gauze, we now introduce a moving flame front that oscillates with speed $u_{s}$ (in the lab frame of reference) around the position $x=b$ inside the duct. We do this because systems with non-small mean flow Mach numbers are more likely to contain flames than heated gauzes. Following the formalism

developed by Strobio Chen et al. [26], and assuming that the flame is anchored (i.e $\bar{u}_{s}=0$ ), the new set of jump conditions reads:

$$
\begin{aligned}
{\left[\rho^{\prime} \bar{u}+\bar{\rho} u^{\prime}\right]_{b^{-}}^{b^{+}} } & =u_{s}^{\prime}\left(\bar{\rho}_{2}-\bar{\rho}_{1}\right) \\
{\left[p^{\prime}+2 \bar{\rho} \bar{u} u^{\prime}+\rho^{\prime} \bar{u}^{2}\right]_{b^{-}}^{b^{+}} } & =0 \\
{\left[\frac{\gamma}{\gamma-1}\left(p^{\prime} \bar{u}+\bar{p} u^{\prime}\right)+\frac{3}{2} \bar{\rho} \bar{u}^{2} u^{\prime}+\frac{1}{2} \rho^{\prime} \bar{u}^{3}\right]_{b^{-}}^{b^{+}} } & =q^{\prime}+u_{s}^{\prime}\left(\frac{\bar{p}_{2}-\bar{p}_{1}}{\gamma-1}+\frac{1}{2} \bar{\rho}_{1} \bar{u}_{1}\left(\bar{u}_{2}-\bar{u}_{1}\right)\right) .
\end{aligned}
$$

The unsteady heat release model is again defined as an $n-\tau$ model proportional to the velocity upstream of the flame, resembling a perfectly premixed flame:

$$
\frac{q^{\prime}}{\bar{q}}=k \frac{u^{\prime}\left(b^{-}, t-\tau\right)}{\bar{u}_{1}}
$$

where $k$ is the flame interaction index and $\tau$ the time delay. To compute $u_{s}^{\prime}$ we follow the approach of Bloxsidge et al. [27]. They consider a 2D flame inside a finite control volume. In the limit of a compact flame (i.e the length of the control volume vanishes) their formalism recovers Eqs. (49) and gives the following expression for the unsteady flame velocity:

$$
u_{s}^{\prime}=\bar{u}_{1}\left(\frac{\rho^{\prime}\left(b^{-}\right)}{\bar{\rho}_{1}}+\frac{u^{\prime}\left(b^{-}\right)}{\bar{u}_{1}}-\frac{q^{\prime}}{\bar{q}}\right)
$$

\subsection{Decoupling the system into Riemann invariants}

Due to convection effects, the continuity and energy equation no longer represent the same dynamics. This is shown by decoupling the system of equations governing the the ducts $(\S 2.2)$, reading $\boldsymbol{V}_{t}+\Lambda \boldsymbol{V}_{x}=\mathbf{0}$ :

$$
\left[\begin{array}{l}
\mathcal{R} \\
\mathcal{U} \\
\mathcal{P}
\end{array}\right]_{t}+\left[\begin{array}{ccc}
\bar{u} & 0 & 0 \\
0 & \bar{c}+\bar{u} & 0 \\
0 & 0 & -(\bar{c}-\bar{u})
\end{array}\right]\left[\begin{array}{l}
\mathcal{R} \\
\mathcal{U} \\
\mathcal{P}
\end{array}\right]_{x}=\mathbf{0}
$$


The solutions are the known forward, $f$, and backward, $g$, acoustic waves, which travel at speeds $\bar{c}+\bar{u}$ and $\bar{c}-\bar{u}$, respectively. In addition, there exists an entropy wave, $\alpha$, convected with the mean flow at speed $\bar{u}$ [see, e.g, 28, 29, 30]. The solutions for the original variables can be expressed as:

$$
\begin{aligned}
& p^{\prime}(x, t)=f\left(t-\frac{x}{\bar{c}+\bar{u}}\right)+g\left(t+\frac{x}{\bar{c}-\bar{u}}\right), \\
& u^{\prime}(x, t)=\frac{1}{\bar{\rho} \bar{c}}\left(f\left(t-\frac{x}{\bar{c}+\bar{u}}\right)-g\left(t+\frac{x}{\bar{c}-\bar{u}}\right)\right), \\
& \rho^{\prime}(x, t)=\frac{1}{\bar{c}^{2}}\left(f\left(t-\frac{x}{\bar{c}+\bar{u}}\right)+g\left(t+\frac{x}{\bar{c}-\bar{u}}\right)\right)+\alpha\left(t-\frac{x}{\bar{u}}\right) .
\end{aligned}
$$

In summary, the mean flow causes corrections to the travelling speeds of the acoustic waves and the appearance of an entropy wave, which convects with the mean flow speed. After assuming a choked end due to a compact nozzle in the outlet, the network model is detailed in Fig. 6.

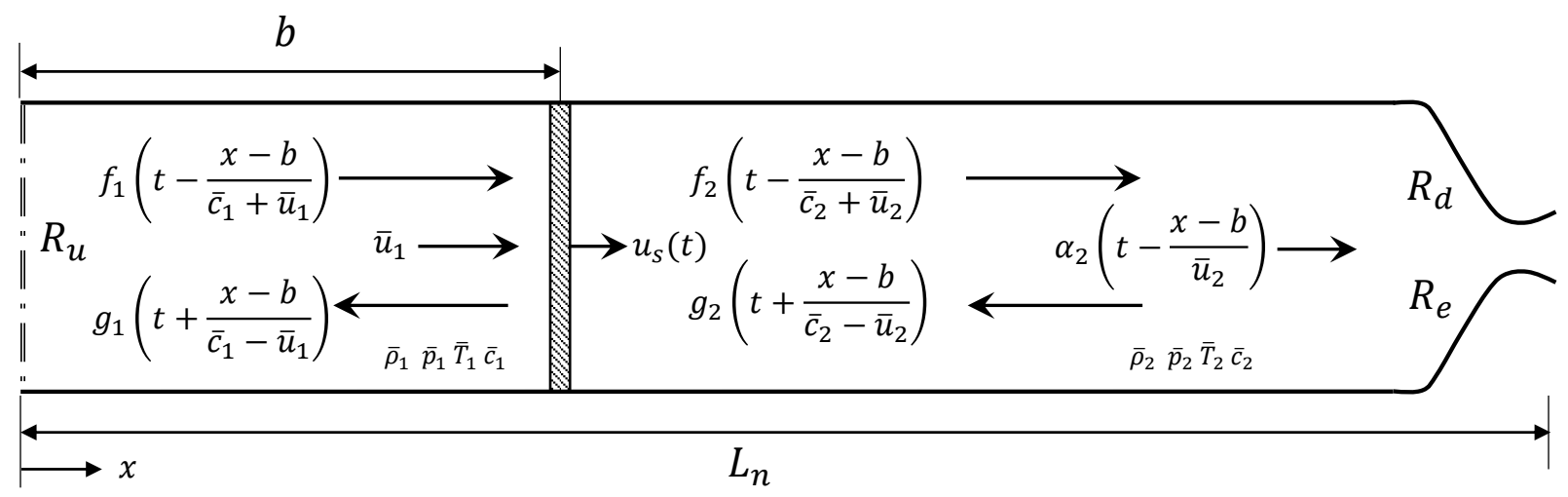

Figure 6: Network model setup considering a mean flow $\bar{u}$ and a choked end. Compared to Fig. 1, this model includes corrections for the propagation speeds of the waves, and an entropy wave generated after the flame. The downstream end is now chocked by a compact nozzle. The reflection coefficient $R_{e}$ represents the strength of the acoustic wave generated by the entropy wave $\alpha_{2}$.

\subsection{Nonlinear eigenvalue problem}

The boundary condition for the upstream acoustic waves defined through the reflection coefficient from Eq. (15) remains almost unaltered. Only the time delay associated with the length of the upstream duct changes to $\tau_{u}=2 b \bar{c}_{1} /\left(\bar{c}_{1}^{2}-\bar{u}_{1}^{2}\right)$. The downstream boundary is now modelled as a choked outlet. Hence, according to Marble and Candel [31], the following boundary condition must be satisfied:

$$
2 \frac{u^{\prime}}{\bar{u}}+\frac{\rho^{\prime}}{\bar{\rho}}-\frac{p^{\prime}}{\bar{p}}=0
$$

this condition allows acoustic fluctuations to be generated from the acceleration of the entropy waves. Thus:

$$
G_{2}(s)=R_{d} F_{2}(s) \mathrm{e}^{-s \tau_{d}}+R_{e} \mathcal{A}_{2}(s) \mathrm{e}^{-s \tau_{e}}
$$


with reflection coefficients depending on the Mach number $M=\bar{u} / \bar{c}$ :

$$
\begin{aligned}
R_{d} & =\frac{1-\frac{1}{2}(\gamma-1) M_{2}}{1+\frac{1}{2}(\gamma-1) M_{2}} & \tau_{d} & =2\left(L_{n}-b\right) \bar{c}_{2} /\left(\bar{c}_{2}^{2}-\bar{u}_{2}^{2}\right), \\
R_{e} & =\frac{\frac{1}{2} M_{2}}{1+\frac{1}{2}(\gamma-1) M_{2}} & \tau_{e} & =\frac{\left(L_{n}-b\right) \bar{c}_{2}}{\bar{u}_{2}\left(\bar{c}_{2}-\bar{u}_{2}\right)} .
\end{aligned}
$$

Entropy fluctuations are often considered as convected hot spots generated by the flame. Therefore they are set to zero at the inlet, $\alpha_{1}=0$. Following a similar approach as in $\S 2.3$ we create an eigenvalue problem: $\mathrm{L}(s) \hat{\boldsymbol{q}}=\mathbf{0} . \mathrm{L}(s)$ is a matrix that includes the effects of the mean flow, $\bar{u}$, through the Mach number, (the components are shown in Appendix B). The vector $\hat{\boldsymbol{q}}=\left[\begin{array}{lll}G_{1} & F_{2} & \mathcal{A}_{2}\end{array}\right]^{T}$ now contains the relative amplitudes between the acoustic and entropy waves. The problem is again nonlinear in the eigenvalue $s$. The eigenvalues and eigenvectors are numerically computed as in $\S 2.3$.

Most of the analysis to obtain the adjoint equations and eigenvalue drift formulae in the zero Mach number case can be extended to the case with a mean flow. Therefore, only a few remarks about the methods are made in the following section.

\subsection{Continuous adjoint approach}

Following the same approach as in section $\S 2.4$, the adjoint variables for the governing equations (48) are $\hat{\rho}^{+}(x), \hat{u}^{+}(x), \hat{p}^{+}(x)$ and for the jump conditions (49) are $\hat{f}^{+}, \hat{g}^{+}, \hat{h}^{+}$. The Lagrangian functional becomes:

$$
\mathcal{L} \equiv s-\left\langle\hat{\rho}^{+}, E_{1}\right\rangle-\left\langle\hat{u}^{+}, E_{2}\right\rangle-\left\langle\hat{p}^{+}, E_{3}\right\rangle-\left\{\hat{f}^{+}, J_{1}\right\}-\left\{\hat{g}^{+}, J_{2}\right\}-\left\{\hat{h}^{+}, J_{3}\right\} .
$$

As explained previously, the derivatives of the Lagrangian with respect to the adjoint variables produce the direct equations. The derivatives of the Lagrangian with respect to the direct variables, after integration by parts, produce the adjoint equations:

$$
\begin{array}{r}
-s^{*} \hat{\rho}^{+}+\bar{u} \frac{\mathrm{d} \hat{\rho}^{+}}{\mathrm{d} x}=0, \\
-s^{*} \bar{\rho} \hat{u}^{+}+\bar{\rho} \bar{u} \frac{\mathrm{d} \hat{u}^{+}}{\mathrm{d} x}+\bar{\rho} \frac{\mathrm{d} \hat{\rho}^{+}}{\mathrm{d} x}+\gamma \bar{p} \frac{\mathrm{d} \hat{p}^{+}}{\mathrm{d} x}=0, \\
-s^{*} \hat{p}^{+}+\bar{u} \frac{\mathrm{d} \hat{p}^{+}}{\mathrm{d} x}+\frac{\mathrm{d} \hat{u}^{+}}{\mathrm{d} x}=0,
\end{array}
$$

along with the relationship between adjoint variables

$$
\begin{aligned}
& \hat{f}^{+}=\hat{\rho}^{+}\left(b^{+}\right)-\bar{u}_{2} \hat{u}^{+}\left(b^{+}\right)+\frac{1}{2}(\gamma-1) \bar{u}_{2}^{2} \hat{p}^{+}\left(b^{+}\right), \\
& \hat{g}^{+}=\hat{u}^{+}\left(b^{+}\right)-(\gamma-1) \bar{u}_{2} \hat{p}^{+}\left(b^{+}\right), \\
& \hat{h}^{+}=(\gamma-1) \hat{p}^{+}\left(b^{+}\right),
\end{aligned}
$$


and the adjoint jump conditions

$$
\begin{aligned}
{\left[\hat{\rho}^{+}-\bar{u} \hat{u}^{+}+\frac{1}{2}(\gamma-1) \bar{u}^{2} \hat{p}^{+}\right]_{1}^{2} } & =-\left(\bar{c}_{1}^{2}-\bar{u}_{1}^{2}\right) \hat{u}_{s}^{+}-\frac{1}{2}(\gamma+1) \bar{u}_{1}^{2} \hat{q}_{s}^{+}, \\
{\left[\hat{u}^{+}-(\gamma-1) \bar{u} \hat{p}^{+}\right]_{1}^{2} } & =\gamma \bar{u}_{1} \hat{q}_{s}^{+}, \\
{\left[\hat{p}^{+}\right]_{1}^{2} } & =-\hat{q}_{s}^{+},
\end{aligned}
$$

where

$$
\begin{aligned}
& \hat{u}_{s}^{+}=\hat{a}_{1}^{+}+\left(\frac{\bar{p}_{2}-\bar{p}_{1}}{\gamma-1}+\frac{1}{2} \bar{\rho}_{1} \bar{u}_{1}\left(\bar{u}_{2}-\bar{u}_{1}\right)\right) \hat{a}_{2}^{+}, \\
& \hat{q}_{s}^{+}=\left(\frac{\bar{q}}{\bar{u}_{1}} \hat{a}_{2}^{+}-\hat{u}_{s}^{+}\right) k \mathrm{e}^{-s^{*} \tau}, \\
& \hat{a}_{1}^{+}=\frac{\left(\bar{\rho}_{2}-\bar{\rho}_{1}\right)}{\bar{\rho}_{1}\left(\bar{c}_{1}^{2}-\bar{u}_{1}^{2}\right)}\left(\hat{\rho}^{+}\left(b^{+}\right)-\bar{u}_{2} \hat{u}^{+}\left(b^{+}\right)+\frac{1}{2}(\gamma-1) \bar{u}_{2}^{2} \hat{p}^{+}\left(b^{+}\right)\right), \\
& \hat{a}_{2}^{+}=\frac{(\gamma-1) \hat{p}^{+}\left(b^{+}\right)}{\bar{\rho}_{1}\left(\bar{c}_{1}^{2}-\bar{u}_{1}^{2}\right)} .
\end{aligned}
$$

The term $\hat{u}_{s}^{+}$is the adjoint equivalent of the flame speed in the lab frame of reference. Physically, it gives the response of the thermoacoustic mode to harmonic forcing of the flame speed. The boundary terms are:

$$
\hat{\rho}^{+*} \bar{u} \hat{\rho}+\hat{\rho}^{+*} \bar{\rho} \hat{u}+\hat{u}^{+*} \bar{\rho} \bar{u} \hat{u}+\hat{u}^{+*} \hat{p}+\hat{p}^{+*} \bar{u} \hat{p}+\hat{p}^{+*} \gamma \bar{p} \hat{u}=0 .
$$

Finally, the derivative with respect to the eigenvalue provides the normalization condition:

$$
\left\langle\hat{\rho}^{+}, \hat{\rho}\right\rangle+\left\langle\hat{u}^{+}, \bar{\rho} \hat{u}\right\rangle+\left\langle\hat{p}^{+}, \hat{p}\right\rangle+\left\{\hat{f}^{+}, N_{m} k \tau \hat{u}\left(b^{-}\right) \mathrm{e}^{-s \tau}\right\}+\left\{\hat{h}^{+}, N_{e} k \tau \hat{u}\left(b^{-}\right) \mathrm{e}^{-s \tau}\right\}=1,
$$

where $N_{m} \equiv \bar{\rho}_{1}-\bar{\rho}_{2}$ and $N_{e} \equiv \bar{q} / \bar{u}_{1}-\left(\left(\bar{p}_{2}-\bar{p}_{1}\right) /(\gamma-1)+\frac{1}{2} \bar{\rho}_{1} \bar{u}_{1}\left(\bar{u}_{2}-\bar{u}_{1}\right)\right)$. Decoupling the system of mean flow adjoint equations (57) as in $\S 2.2$, yields exactly the same system as that presented in Eq. (52). That is, two adjoint acoustic waves convected at speeds $\bar{c}+\bar{u}$ and $\bar{c}-\bar{u}$ and an adjoint entropy wave convected at the mean speed $\bar{u}$. This shows that, even with a mean flow, the equations governing duct acoustics remain self-adjoint. Adding the flame or the boundary conditions, however, make the system non-self-adjoint. In the direct eigenvalue problem $\S 5.3$ we assumed no entropy waves upstream of the flame. Hence, Eq. (60) provides the upstream adjoint reflection coefficient relationship at the inlet:

$$
R_{u}^{+}=\frac{1}{R_{u}{ }^{*}} \frac{1-M_{1}}{1+M_{1}}
$$

then, as before, the upstream adjoint boundary condition is:

$$
G_{1}^{+}=R_{u}^{+-1} F_{1}^{+} \mathrm{e}^{-s^{*} \tau_{u}}
$$

Due to the choked end, the downstream acoustic and entropy waves are reflected as an acoustic wave. In the adjoint problem, however, the outgoing adjoint acoustic wave at the choked end creates both a backward adjoint acoustic wave and a backward adjoint entropy wave. This is because 
the direct equations propagate quantities forwards in time, while the adjoint equations propagate receptivities backwards in time. Equation (60) gives the reflection coefficient relationships at the outlet:

$$
\begin{aligned}
R_{d}^{+} & =\frac{1}{R_{d}} \frac{1+M_{2}}{1-M_{2}}, \\
R_{e}^{+} & =\frac{1}{R_{e}} \frac{M_{2}}{2\left(M_{2}-1\right)} .
\end{aligned}
$$

With these expressions, the downstream adjoint boundary conditions are obtained:

$$
\begin{aligned}
& F_{2}^{+}=R_{d}^{+-1} G_{2}^{+} \mathrm{e}^{-s^{*} \tau_{d}}, \\
& A_{2}^{+}=R_{e}^{+-1} G_{2}^{+} \mathrm{e}^{-s^{*} \tau_{e}} .
\end{aligned}
$$

The procedure to obtain the adjoint eigenvalue problem, and the eigenvalue drift for the base state and feedback sensitivities, remains the same as in $\S 2.4 .2$.

\subsection{Discrete adjoint approach}

Since the mean flow nonlinear eigenvalue problem has the same form as that in the zero Mach number limit (17), the adjoint problem (41) and eigenvalue drift formula (42) remain unaltered. Thus, the matrix $\delta \mathrm{L}$ associated with the base state sensitivity is again provided by equation (43). The feedback sensitivity can now consider the effects of mass injection, $\mathcal{M}$, and feedback devices can be extended to include density fluctuations. Hence, instead of four possible feedback mechanisms there are now nine. The procedure to create the matrix $\delta \mathrm{L}$ is as described in $\S$ 2.5.2. However, the inclusion of entropy waves makes the sets of jump relations change slightly. The jump conditions upstream of the flame $(x=a)$ become:

$$
\begin{aligned}
\bar{u}_{1}\left(\rho_{i i}^{\prime}-\rho_{i}^{\prime}\right)+\bar{\rho}_{1}\left(u_{i i}^{\prime}-u_{i}^{\prime}\right) & =\mathcal{M}_{p_{1}} p_{i}^{\prime}+\mathcal{M}_{u_{1}} u_{i}^{\prime}+\mathcal{M}_{r_{1}} \rho_{i}^{\prime}, \\
\bar{\rho}_{1} \bar{u}_{1}\left(u_{i i}^{\prime}-u_{i}^{\prime}\right)+\left(p_{i i}^{\prime}-p_{i}^{\prime}\right) & =\mathcal{F}_{p_{1}} p_{i}^{\prime}+\mathcal{F}_{u_{1}} u_{i}^{\prime}+\mathcal{F}_{r_{1}} \rho_{i}^{\prime}, \\
\bar{u}_{1}\left(p_{i i}^{\prime}-p_{i}^{\prime}\right)+\gamma \bar{p}_{1}\left(u_{i i}^{\prime}-u_{i}^{\prime}\right) & =\mathcal{E}_{p_{1}} p_{i}^{\prime}+\mathcal{E}_{u_{1}} u_{i}^{\prime}+\mathcal{E}_{r_{1}} \rho_{i}^{\prime},
\end{aligned}
$$

while the jump conditions downstream of the flame $(x=c)$ become

$$
\begin{aligned}
\bar{u}_{2}\left(\rho_{i v}^{\prime}-\rho_{i i i}^{\prime}\right)+\bar{\rho}_{2}\left(u_{i v}^{\prime}-u_{i i i}^{\prime}\right) & =\mathcal{M}_{p_{2}} p_{i v}^{\prime}+\mathcal{M}_{u_{2}} u_{i v}^{\prime}+\mathcal{M}_{r_{2}} \rho_{i v}^{\prime} \\
\bar{\rho}_{2} \bar{u}_{2}\left(u_{i v}^{\prime}-u_{i i i}^{\prime}\right)+\left(p_{i v}^{\prime}-p_{i i i}^{\prime}\right) & =\mathcal{F}_{p_{2}} p_{i v}^{\prime}+\mathcal{F}_{u_{2}} u_{i v}^{\prime}+\mathcal{F}_{r_{2}} \rho_{i v}^{\prime} \\
\bar{u}_{2}\left(p_{i v}^{\prime}-p_{i i i}^{\prime}\right)+\gamma \bar{p}_{2}\left(u_{i v}^{\prime}-u_{i i i}^{\prime}\right) & =\mathcal{E}_{p_{2}} p_{i v}^{\prime}+\mathcal{E}_{u_{2}} u_{i v}^{\prime}+\mathcal{E}_{r_{2}} \rho_{i v}^{\prime}
\end{aligned}
$$

where the term $\mathcal{E} \equiv \mathcal{Q}+\bar{c}^{2} \mathcal{M}$ arises due to the use of the continuity equation to express the energy equation in terms of pressure. Some features of the wave-forms together with their first variations $(\delta f, \delta g, \delta \alpha)$ are that the acoustic waves $(f$ and $g)$ are affected by mass, momentum and energy addition and are accompanied by a $1 /(2(M \pm 1))$ factor, while the entropy waves $(\alpha)$ are only affected by energy addition and accompanied by a $1 / M$ factor. 


\section{Results for a choked combustor with mean flow and a moving flame front}

\subsection{Thermoacoustic configuration}

For this flow configuration, we use the same properties for the air and inlet conditions as in $\S$ 4.1. However, we now assume a mean flow Mach number of $M_{1}=5.00 \times 10^{-2}$, which requires an inlet steady flow of $\bar{u}_{1}=17.4 \mathrm{~ms}^{-1}$. The flame causes a jump in temperature reaching $\bar{T}_{2}=1500 \mathrm{~K}$ in the downstream side, which corresponds to a mean steady heat release of $\bar{q}=24.7 \mathrm{MWm}^{-2}$, which is similar to the heat release of an intermediate scale rig [32]. The flame interaction index is chosen to be $k=1.00$ and the time delay $\tau=1.00 \times 10^{-3} \mathrm{~s}$. The relevant eigenvalue becomes $s_{1}=(145.2+2 \pi \times 184.4 i) \mathrm{rad} \mathrm{s}^{-1}$ or $\tilde{s}_{1}=0.4180+3.336 i$.

\subsection{Stability and sensitivity analysis}

The mode shapes for this configuration are shown in Fig. 7. Pressure (Fig. 7a) displays the open ended inlet condition and a jump caused by the combined effect of the mean flow and unsteady heat release (49). Velocity (Fig. 7b) has a jump at the flame location and shows the behaviour of a nearly closed end due to the choked boundary condition. In the region upstream of the flame, density (Fig. 7c) shows a similar behaviour as the pressure (scaled by a $1 / \bar{c}_{1}^{2}$ factor). After the flame, density is the only mode shape that contains the influence of both acoustic and entropy waves. Small amplitude entropy waves appear in the downstream region because the acoustic fluctuations change the temperature upstream, (and the model assumes that the mean heat release is kept constant) [26]. The generated hot spots are accelerated at the nozzle and converted into indirect acoustic waves. The ratio between the amplitudes of the indirect acoustic wave and the direct acoustic wave is $0.53 \%$, which directly correlates to the small contribution of indirect noise in perfectly premixed flames. For comparison, in the same configuration a heat source at rest $\left(u_{s}^{\prime}=0\right)$ with the same heating capacity, such as a gauze heater, gives a ratio of $20.70 \%$.

Adjoint entropy waves appear upstream and downstream of the flame in the receptivity of the continuity equation $\left(\hat{\rho}^{+}\right.$in Fig. 7c) and as oscillations in the receptivity of the energy equation $\left(\hat{p}^{+}\right.$in Fig. 7a). In the latter, they appear in the upstream region mainly due to the effect of the flame. They appear in the downstream region due to the choked end as explained in $\S$ 5.4. This physically means that to produce a change in the generation of entropy waves through the unsteady flame speed, we need to force the energy equation before the flame, as intuitively expected. Forcing the energy equation after the flame changes the intensity with which the entropy waves interact with the choked end.

For this configuration, the base state sensitivities for different flame positions are plotted in Fig. 8. (The choked end does not allow a variation of the reflection coefficients in the downstream end, and its sensitivity is thus omitted.) The upstream reflection coefficient shows a similar behaviour to the case without a mean flow. The sensitivity of the flame interaction index shows that any increase in its value when the flame is in the first fifth of the duct will stabilize the system but will destabilize the system everywhere else. On the other hand the frequency is largely affected when the flame is located in the first half of the duct, when compared to the second half. This is because the unsteady heat release is proportional to velocity and its magnitude is reduced towards the chocked end. The sensitivity of the time delay has the strongest growth rate shift 
(a)

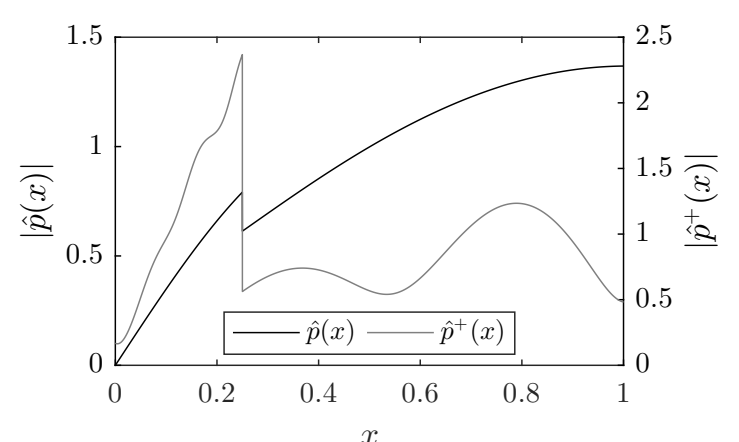

(b)

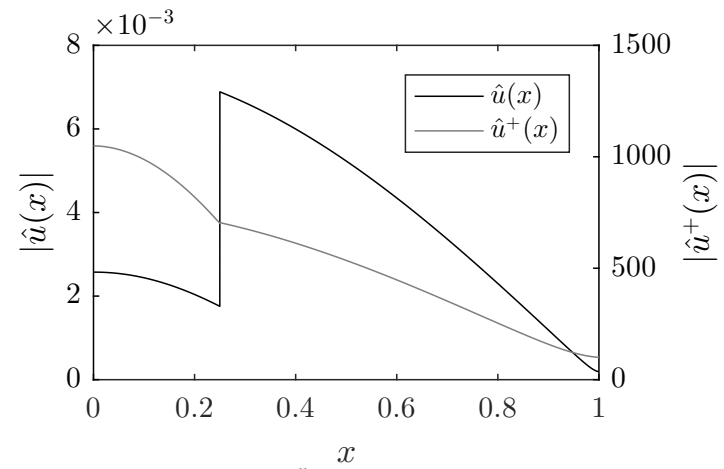

(c)

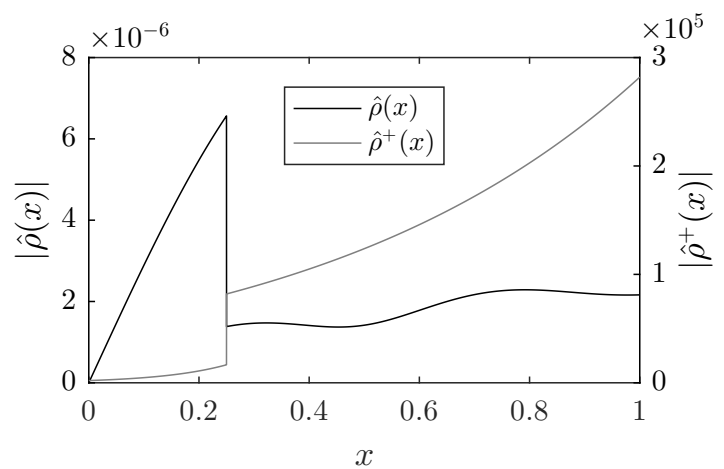

Figure 7: Mode shapes for the direct and adjoint variables. (a), (b) and (c) show the relative amplitudes between pressure, velocity and density fluctuations respectively (i.e. $\hat{p}(x)=|\hat{p}(x)| \mathrm{e}^{i \theta}$ ) as well as the receptivity to the energy, momentum and continuity equations respectively (computed using the continuous adjoint approach).

from the other two variables. Any increase will destabilize the system as soon as the flame is not located near the inlet of the duct, and the effect is similar for the frequency shift as well.

(a)

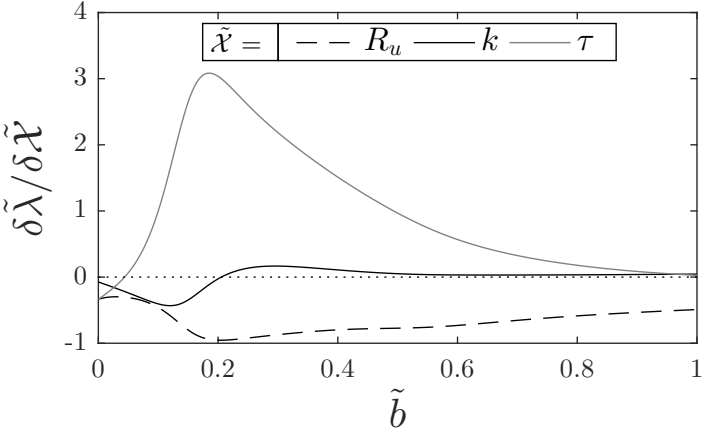

(b)

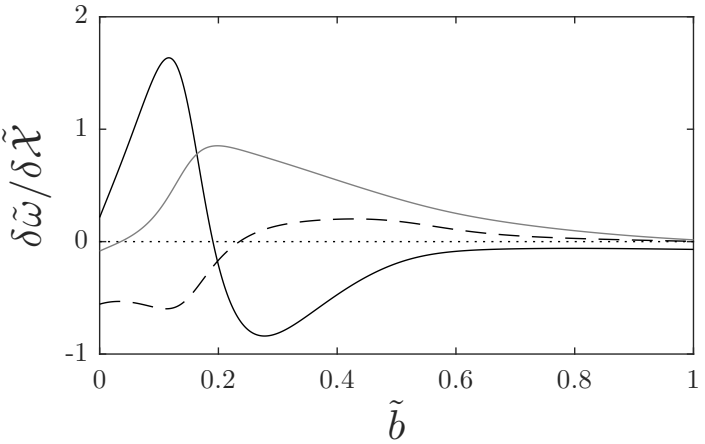

Figure 8: Growth rate (a) and angular frequency (b) shifts of the base state variables, denoted by $\tilde{\mathcal{X}}$, for different positions of the flame, $\tilde{b}$, along the duct length considering a mean flow, $\bar{u}$, and a moving flame front.

For the feedback sensitivity, we first analyse the mechanisms that cause mass injection (row 1 of Fig. 9). For this system any feedback mechanism that causes mass addition in phase with $\hat{p}, \hat{u}$, or $\hat{\rho}$, will destabilize the system, as seen by the growth rate shifts. The most physical mechanism is represented by feedback from pressure Fig. 9a, which models the effect of positioning a Helmholtz 
resonator in the duct. (The phase of the resultant mass addition depends on the oscillation frequency relative to the Helmholtz resonator frequency). In a system with a choked end, the most influential position to locate such a device is towards the exit $x=0.96$, which is not quite the antinode of the resonant mode.

The mechanisms that cause momentum addition in phase with $\hat{p}, \hat{u}$, or $\hat{\rho}$, are shown in row 2 of Fig. 9. The most physical mechanism would be the drag device from Fig. 9e, which forces the flow $\pi$ out of phase with (i.e. in the opposite direction to) the local velocity, and is strongly stabilizing. This can be seen by taking the negative of the growth rate (continuous black line or filled dots) in Fig. 9e.

The mechanisms that cause heat addition in phase with $\hat{p}, \hat{u}$, or $\hat{\rho}$, are shown in row 3 of Fig. 9. In all of these mechanisms there are oscillations upstream and downstream of the flame. These are caused by the entropy waves and their contribution, after being accelerated at the choked end, as acoustic waves. If entropy waves were allowed to convect away from the system, the mechanisms would be similar to those for mass injection, with oscillations only upstream of the flame. For example, any mechanism that causes increased heat input in phase with the pressure will destabilize the system, as is the case for solid rocket propellants.

In a choked nozzle two upstream-propagating acoustic waves are generated at the throat: one by the entropy wave and one by the downstream-propagating acoustic wave. If the superposition of the two upstream propagating waves is constructive, the feedback mechanisms will present similar shapes but with larger amplitudes. If the superposition of waves is destructive, the feedback mechanisms will present similar shapes but smaller amplitudes. For this particular configuration the latter case is present, therefore the feedback devices are weakened by the indirect noise. Investigating whether this is a general result or a specific feature of the considered configuration is left for future studies.

\section{Conclusions}

The main goal of this paper is to extend adjoint sensitivity analysis in thermoacoustics to nonlinear eigenvalue problems derived from wave-based network models. These models can tackle mean flows and are more versatile than Galerkin methods, for which adjoint methods have already been developed.

Two thermoacoustic models are developed. The first mode has a heating gauze and a zero Mach number. The second model, which is more general, has a moving flame front and a mean flow. In each model, the system is decoupled into acoustic (and entropic when $M \neq 0$ ) waves, creating a nonlinear eigenvalue problem. The adjoint eigenvalue problem is set up with two different methods: the continuous adjoint and the discrete adjoint approach. In the continuous approach, the adjoint equations are derived by a Lagrangian formalism. We mathematically show that the adjoint variables are governed by a wave equation. The adjoint equations are then solved in the same way as the direct equations, by obtaining the eigenvalues and right eigenvectors of the adjoint matrix. In the discrete approach, the adjoint eigenvector is simply the left eigenvector of the direct matrix. For the wave-based model in this paper, the continuous approach provides greater physical understanding in terms of receptivity because the adjoint spatial functions are known. 


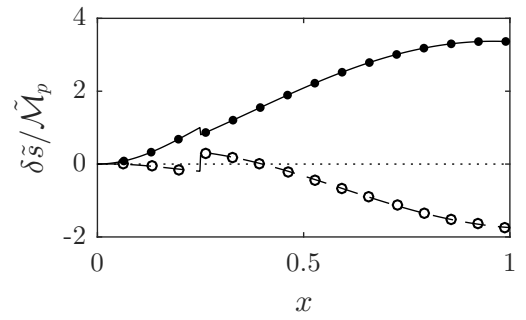

(a)

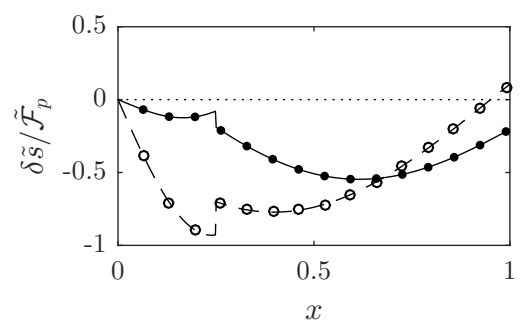

(d)

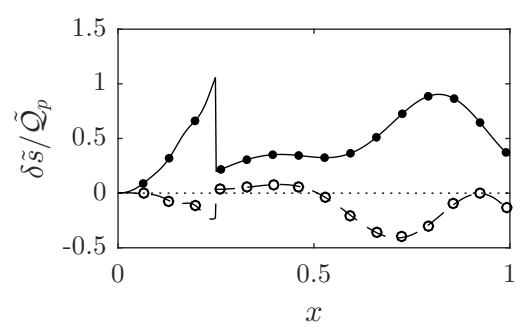

(g)

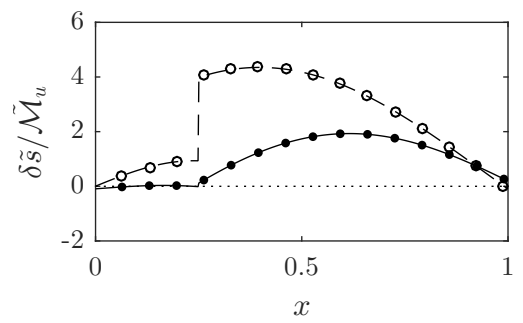

(b)

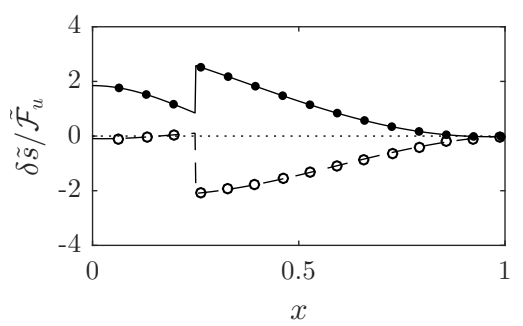

(e)

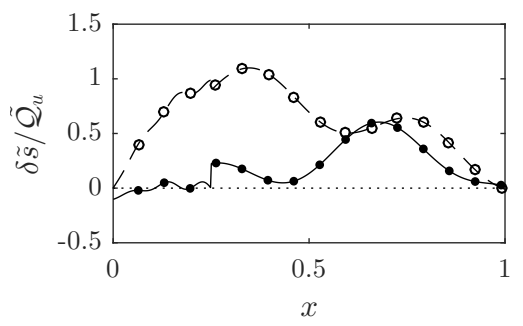

(h)

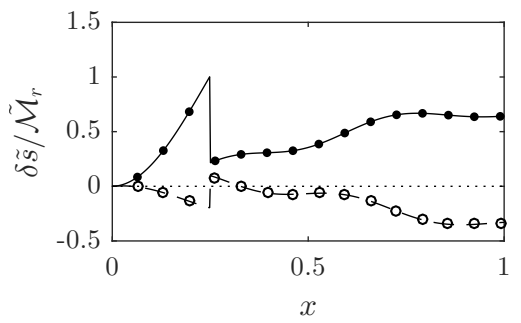

(c)

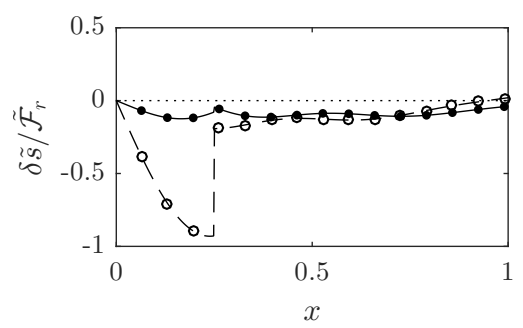

(f)

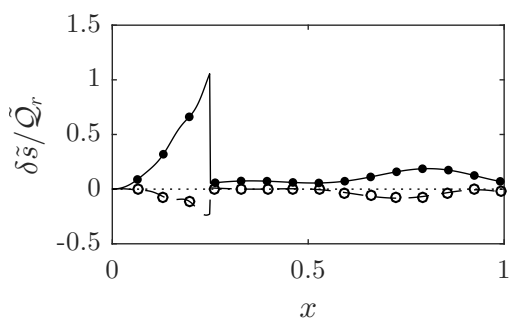

(i)

Figure 9: Growth rate shift, $\delta \tilde{\lambda}$ denoted by -, •, and angular frequency shift, $\delta \tilde{\omega}$ denoted by - - - , ०, for each feedback mechanism. The rows display the continuity, momentum and energy equations, while the columns represent feedback from pressure, velocity and density respectively. The continuous and dashed lines (-,- - -) represent one calculation via the adjoint method while the circles $(\bullet, \circ)$ represent 15 computations via the finite difference method.

This analysis shows that the equations governing duct acoustics, without considering boundary conditions, are self-adjoint, with or without a mean flow. We also show that the system with no unsteady heat release and reflection coefficients with magnitude different from 1 is selfadjoint under the transformation $t \rightarrow-t$, which is equivalent to saying that forward-propagating waves become backward-propagating waves and vice-versa. The thermoacoustic system becomes non-self-adjoint when there is velocity-coupled unsteady heat release, in agreement with findings available in the literature. By combining the eigenfunctions from the direct and adjoint systems, we obtain the base state sensitivities and feedback sensitivities with cheap computations. The base state sensitivity is easier to implement with the discrete approach, and the feedback sensitivity is easier to implement with the continuous approach. Both adjoint methods provide the same results, which are validated against finite-difference calculations to machine precision. The base state sensitivity provides the gradient of the eigenvalue with respect to any parameter of the 
model in a single calculation. Hence, it reveals how to stabilize (or destabilize) the the system. The feedback sensitivity provides the gradient of the eigenvalue with respect to linear feedback mechanisms. In this paper, it is obtained for co-located sensors and actuators.

The base state sensitivity analysis shows that the most influential stability parameter is the time delay between velocity and heat release. In the zero Mach number case, the feedback sensitivity shows the influence of devices that react to the velocity or pressure fluctuations and force the momentum or energy equations. For the non-zero Mach number case, the feedback sensitivity also shows the influence of devices that react to the density and devices that force the mass equation. However, density-driven devices are difficult to devise and their influence depends on the strength of the entropy waves, and consequently on the flame model. For a perfectly premixed flame they display very small eigenvalue shifts in the downstream region due to the small entropy waves. As a result, they would probably be ineffective in practice. The choked outlet in this configuration accelerates the hot spots producing indirect noise, which in turn reduces the overall amplitude of all the feedback mechanisms.

The optimal position to locate a feedback device is given by the product of the direct and adjoint eigenfunctions. In a self-adjoint system, the adjoint eigenfunction is identical to the direct eigenfunction, so the optimal position can be determined from the direct eigenfunctions alone. In a non-self-adjoint system, however, the adjoint eigenfunction differs from the direct eigenfunction and the optimal placement is altered accordingly. This needs to be taken into account in thermoacoustic systems, which are non-self-adjoint. For example, this information can aid the design of retrofitted devices such as Helmholtz resonators.

In summary, this paper develops and applies adjoint methods to wave-based thermoacoustic network models, which are used in industrial design of gas turbine engines. The adjoint methods provides sensitivities of the eigenvalues at very little cost. The combination of gradientinformation with optimization routines could improve the design to mitigate (or delay the onset of) thermoacoustic instabilities in gas turbines.

\section{Acknowledgments}

J.G.A is grateful to Alessandro Orchini for fruitful discussions and comments on this paper, and thankfully acknowledges CONACyT and Cambridge Trust for funding this project. L.M. gratefully acknowledges the financial support from the Royal Academy of Engineering Research Fellowships scheme.

\section{Appendix A. Computing matrix $\delta \mathrm{L}$}

Once the boundary conditions have been applied, by taking the Laplace transform of the perturbed wave solutions (i.e Eq. 46) we obtain:

$$
\begin{aligned}
& \mathscr{L}\left(f_{i i}\right)=F_{i i}=G_{1}\left(R_{u} \mathrm{e}^{-s \tau_{u}}+E_{F_{1}}\right), \\
& \mathscr{L}\left(g_{i i}\right)=G_{i i}=G_{1}\left(1+E_{G_{1}}\right), \\
& \mathscr{L}\left(f_{i i i}\right)=F_{i i i}=F_{2}\left(1+E_{F_{2}}\right), \\
& \mathscr{L}\left(g_{i i i}\right)=G_{i i i}=F_{2}\left(R_{d} \mathrm{e}^{-s \tau_{d}}+E_{G_{2}}\right),
\end{aligned}
$$


where

$$
\begin{aligned}
& E_{F_{1}}=\frac{1}{2}( \mathcal{F}_{p_{1}}\left(R_{u} \mathrm{e}^{-s \tau_{u}}+\mathrm{e}^{-s \tau_{a}}\right)+\frac{\mathcal{F}_{u_{1}}}{\bar{\rho}_{1} \bar{c}_{1}}\left(R_{u} \mathrm{e}^{-s \tau_{u}}-\mathrm{e}^{-s \tau_{a}}\right) \cdots \\
&\left.+\frac{\mathcal{Q}_{p_{1}}}{\bar{c}_{1}}\left(R_{u} \mathrm{e}^{-s \tau_{u}}+\mathrm{e}^{-s \tau_{a}}\right)+\frac{\mathcal{Q}_{u_{1}}}{\bar{\rho}_{1} \bar{c}_{1}^{2}}\left(R_{u} \mathrm{e}^{-s \tau_{u}}-\mathrm{e}^{-s \tau_{a}}\right)\right) \\
& E_{G_{1}}=\frac{1}{2}( \mathcal{F}_{p_{1}}\left(R_{u} \mathrm{e}^{-s\left(\tau_{u}-\tau_{a}\right)}+1\right)+\frac{\mathcal{F}_{u_{1}}}{\bar{\rho}_{1} \bar{c}_{1}}\left(R_{u} \mathrm{e}^{-s\left(\tau_{u}-\tau_{a}\right)}-1\right) \cdots \\
&\left.-\frac{\mathcal{Q}_{p_{1}}}{\bar{c}_{1}}\left(R_{u} \mathrm{e}^{-s\left(\tau_{u}-\tau_{a}\right)}+1\right)-\frac{\mathcal{Q}_{u_{1}}}{\bar{\rho}_{1} \bar{c}_{1}^{2}}\left(R_{u} \mathrm{e}^{-s\left(\tau_{u}-\tau_{a}\right)}-1\right)\right), \\
& E_{F_{2}}=\frac{1}{2}\left(-\mathcal{F}_{p_{2}}\left(1+R_{d} \mathrm{e}^{-s\left(\tau_{d}-\tau_{c}\right)}\right)-\frac{\mathcal{F}_{u_{2}}}{\bar{\rho}_{2} \bar{c}_{2}}\left(1-R_{d} \mathrm{e}^{-s\left(\tau_{d}-\tau_{c}\right)}\right) \cdots\right. \\
&\left.-\frac{\mathcal{Q}_{p_{2}}}{\bar{c}_{2}}\left(1+R_{d} \mathrm{e}^{-s\left(\tau_{d}-\tau_{c}\right)}\right)-\frac{\mathcal{Q}_{u_{2}}}{\bar{\rho}_{2} \bar{c}_{2}^{2}}\left(1-R_{d} \mathrm{e}^{-s\left(\tau_{d}-\tau_{c}\right)}\right)\right), \\
& E_{G_{2}=} \frac{1}{2}\left(-\mathcal{F}_{p_{2}}\left(\mathrm{e}^{-s \tau_{c}}+R_{d} \mathrm{e}^{-s \tau_{d}}\right)-\frac{\mathcal{F}_{u_{2}}}{\bar{\rho}_{2} \bar{c}_{2}}\left(\mathrm{e}^{-s \tau_{c}}-R_{d} \mathrm{e}^{-s \tau_{d}}\right) \cdots\right. \\
&\left.+\frac{\mathcal{Q}_{p_{2}}}{\bar{c}_{2}}\left(\mathrm{e}^{-s \tau_{c}}+R_{d} \mathrm{e}^{-s \tau_{d}}\right)+\frac{\mathcal{Q}_{u_{2}}}{\bar{\rho}_{2} \bar{c}_{2}^{2}}\left(\mathrm{e}^{-s \tau_{c}}-R_{d} \mathrm{e}^{-s \tau_{d}}\right)\right)
\end{aligned}
$$

where $\tau_{a}=2(b-a) / \bar{c}_{1}$ and $\tau_{c}=2(c-b) / \bar{c}_{2}$. After substitution of the these expressions into the jump conditions (Eq. 6$)$ we get the perturbed problem:

$$
(\mathrm{L}+\delta \mathrm{L})(\hat{\boldsymbol{q}}+\delta \hat{\boldsymbol{q}})=\mathbf{0},
$$

where L and $\hat{\boldsymbol{q}}$ are given by Eq. (17), and $\delta \mathrm{L}$ is:

$$
\delta \mathrm{L}=\left[\begin{array}{cc}
-E_{F_{1}}-E_{G_{1}} & E_{F_{2}}+E_{G_{2}} \\
\left(E_{G_{1}}-E_{F_{1}}\right)\left(1+\frac{\gamma-1}{\gamma \bar{p}_{1}} \beta \mathrm{e}^{-s \tau}\right) & \frac{\bar{c}_{2}}{\bar{c}_{1}}\left(E_{F_{2}}-E_{G_{2}}\right)
\end{array}\right] .
$$

\section{Appendix B. Mean flow matrix L(s)}

The components of the mean flow matrix $\mathrm{L}(s)$ are:

$$
\begin{aligned}
\mathrm{L}_{11} & =\frac{\bar{\rho}_{2}}{\bar{\rho}_{1}}\left(\left(1-M_{1}\right)-R_{u} \mathrm{e}^{-s \tau_{u}}\left(1+M_{1}\right)\right)-\left(\frac{\bar{\rho}_{2}}{\bar{\rho}_{1}}-1\right) k \mathrm{e}^{-s \tau}\left(1-R_{u} \mathrm{e}^{-s \tau}\right) \\
\mathrm{L}_{12} & =\frac{\bar{c}_{1}}{\bar{c}_{2}}\left(\left(M_{2}+1\right)+R_{d} \mathrm{e}^{-s \tau_{d}}\left(M_{2}-1\right)\right) \\
\mathrm{L}_{13} & =\frac{\bar{c}_{1}}{\bar{c}_{2}}\left(M_{2}+R_{e} \mathrm{e}^{-s \tau_{e}}\left(M_{2}-1\right)\right) \\
\mathrm{L}_{21} & =-\left(M_{1}-1\right)^{2}-R_{u} \mathrm{e}^{-s \tau_{u}}\left(M_{1}+1\right)^{2} \\
\mathrm{~L}_{22} & =\left(M_{2}+1\right)^{2}+R_{d} \mathrm{e}^{-s \tau_{d}}\left(M_{2}-1\right)^{2} \\
\mathrm{~L}_{23} & =M_{2}^{2}+R_{e} \mathrm{e}^{-s \tau_{e}}\left(M_{2}-1\right)^{2}
\end{aligned}
$$




$$
\begin{aligned}
\mathrm{L}_{31} & =\frac{1-\gamma M_{1}}{\gamma-1}+\frac{M_{1}^{2}}{2}\left(3-M_{1}\right)-N\left(1-M_{1}-k \mathrm{e}^{-s \tau}\right) \cdots \\
& -R_{u} \mathrm{e}^{-s \tau_{u}}\left(\frac{1+\gamma M_{1}}{\gamma-1}+\frac{M_{1}^{2}}{2}\left(3+M_{1}\right)-N\left(1+M_{1}-k \mathrm{e}^{-s \tau}\right)\right)+k \frac{\bar{q}}{\bar{u}_{1}} \frac{\mathrm{e}^{-s \tau}}{\bar{\rho}_{1} \bar{c}_{1}^{2}}\left(1-R_{u} \mathrm{e}^{-s \tau}\right) \\
\mathrm{L}_{32} & =\frac{\bar{c}_{2}}{\bar{c}_{1}}\left(\frac{1+\gamma M_{2}}{\gamma-1}+\frac{M_{2}^{2}}{2}\left(3+M_{2}\right)-R_{d} \mathrm{e}^{-s \tau_{d}}\left(\frac{1-\gamma M_{2}}{\gamma-1}+\frac{M_{2}^{2}}{2}\left(3-M_{2}\right)\right)\right) \\
\mathrm{L}_{33} & =\frac{1}{2} M_{2}^{3}+R_{e} \mathrm{e}^{-s \tau_{e}}\left(\left(M_{2}-1\right)\left(\frac{1}{\gamma-1}-M_{2}+\frac{M_{2}^{2}}{2}\right)\right)
\end{aligned}
$$

where

$$
N=\frac{1}{2}\left(M_{1}^{2}-M_{2}^{2} \frac{\bar{p}_{2}}{\bar{p}_{1}}\right)+\frac{1-\bar{p}_{2} / \bar{p}_{1}}{\gamma(\gamma-1)}
$$

Note that for simplicity, the amplitude of the entropy wave $\mathcal{A}_{2}$ was multiplied by $1 / \bar{c}_{2}^{2}$ factor.

\section{References}

[1] F. E. C. Culick, Unsteady motions in combustion chambers for propulsion systems, vol. 323, RTO AGARDograph/AG-AVT-039. North Atlantic Treaty Organization (NATO), 2006.

[2] A. P. Dowling, The calculation of thermoacoustic oscillations, Journal of Sound and Vibration 180 (4) (1995) 557-581.

[3] S. R. Stow, A. P. Dowling, Thermoacoustic oscillations in an annular combustor, ASME Turbo Expo (2001) 2001-GT-0037.

[4] A. P. Dowling, A. S. Morgans, Feedback control of combustion oscillations, Annual Review of Fluid Mechanics 37 (1) (2005) 151-182.

[5] A. P. Dowling, S. R. Stow, Acoustic analysis of gas turbine combustors, Journal of Propulsion and Power 19 (5) (2003) 751-764.

[6] D. C. Hill, A theoretical approach for analyzing the restabilization of wakes, in: Presented at AIAA Aersoapce Science Meeting Exhib. 30th, Reno, NV, AIAA Pap., vol. 0067, 1992.

[7] P. Luchini, A. Bottaro, Adjoint equations in stability analysis, Annual Review of Fluid Mechanics 46 (1) (2014) 493-517.

[8] M. P. Juniper, Triggering in the horizontal Rijke tube: non-normality, transient growth and bypass transition, Journal of Fluid Mechanics 667 (2011) 272-308.

[9] L. Magri, M. P. Juniper, Sensitivity analysis of a time-delayed thermo-acoustic system via an adjoint-based approach, Journal of Fluid Mechanics 719 (2013) 183-202.

[10] G. Rigas, N. P. Jamieson, L. K. B. Li, M. P. Juniper, Experimental sensitivity analysis and control of thermoacoustic systems, Journal of Fluid Mechanics 787 (2016) R1 1-11. 
[11] M. P. Juniper, L. Magri, M. Bauerheim, Sensitivity analysis of thermo-acoustic eigenproblems with adjoint methods, Center for Turbulence Research Proceedings of the Summer Program 2014 .

[12] L. Magri, Adjoint methods in thermo-acoustic and combustion instability, Ph.D. thesis, 2015.

[13] L. Magri, M. Bauerheim, M. P. Juniper, Stability analysis of thermo-acoustic nonlinear eigenproblems in annular combustors. Part I. Sensitivity, Journal of Computational Physics 325 (2016) 395-410.

[14] L. M. B. d. C. Campos, Generalized calculus with applications to matter and forces, CRC Press Taylor \& Francis Group, Boca Raton, 1st edn., 2014.

[15] F. Nicoud, L. Benoit, C. Sensiau, T. Poinsot, Acoustic modes in combustors with complex impedances and multidimensional active flames, AIAA Journal 45 (2) (2007) 426-441.

[16] K. Wieczorek, C. Sensiau, W. Polifke, F. Nicoud, Assessing non-normal effects in thermoacoustic systems with mean flow, Physics of Fluids 23 (10).

[17] F. Giannetti, P. Luchini, Structural sensitivity of the first instability of the cylinder wake, Journal of Fluid Mechanics 581 (2007) 167.

[18] L. Magri, M. Bauerheim, F. Nicoud, M. P. Juniper, Stability analysis of thermo-acoustic nonlinear eigenproblems in annular combustors. Part II. Uncertainty quantification., Journal of Computational Physics, under review, arXiv: 1602.08440v1 325 (2016) 395-410.

[19] G. A. Mensah, J. P. Moeck, Assessment of thermoacoustic instabilities based on high-order adjoint perturbation theory, Proceedings of the International Symposium: Thermoacoustic instabilities in gas turbines and rocket engines: industry meets academia (GTRE - 032) (2016) 1-9.

[20] C. F. Silva, T. Runte, W. Polifke, L. Magri, Uncertainty quantification of growth rates of thermoacoustic instability by an adjoint Helmholtz solver, Journal of Engineering for Gas Turbines and Power 139 (1) (2016) 1-11.

[21] L. Crocco, Research on combustion instability in liquid propellant rockets, Symposium (International) on Combustion 12 (1) (1969) 85-99.

[22] L. Magri, M. P. Juniper, Adjoint-based linear analysis in reduced-order thermo-acoustic models, International Journal of Spray and Combustion Dynamics 6 (3).

[23] M. Gunzburger, Introduction into mathematical aspects of flow control and optimization, in: von Karman Institute for Fluid Dynamics, 1997.

[24] J. Aguilar, Sensitivity analysis in low order thermoacoustic networks, Tech. Rep. December, 2015 . 
[25] H. Mangesius, W. Polifke, A discrete-time, state-space approach for the investigation of nonnormal effects in thermoacoustic systems, International Journal of Spray and Combustion Dynamics 3 (4) (2011) 331-350.

[26] L. Strobio Chen, S. Bomberg, W. Polifke, Propagation and generation of acoustic and entropy waves across a moving flame front, Combustion and Flame 166 (2016) 170-180.

[27] G. J. Bloxsidge, A. P. Dowling, P. J. Langhorne, Reheat buzz: an acoustically coupled combustion instability. Part 2. Theory, Journal of Fluid Mechanics 193 (1988) 445-473.

[28] C. S. Goh, A. S. Morgans, The influence of entropy waves on the thermoacoustic stability of a model combustor, Combustion Science and Technology 185 (2) (2013) 249-268.

[29] A. P. Dowling, Y. Mahmoudi, Combustion noise, Proceedings of the Combustion Institute 35 (1) (2015) 65-100.

[30] A. S. Morgans, I. Duran, Entropy noise: A review of theory, progress and challenges, International Journal of spray and combustion synamics 0 (0) (2016) 1-14.

[31] F. Marble, S. Candel, Acoustic disturbance from gas non-uniformities convected through a nozzle, Journal of Sound and Vibration 55 (2) (1977) 225-243.

[32] T. C. Lieuwen, V. Yang (Eds.), Combustion instabilities in gas turbine engines: operational experience, fundamental mechanisms, and modeling, AIAA, 2005. 